Article

\title{
Atmospheric Rivers, Floods and the Water Resources of California
}

\section{Michael D. Dettinger ${ }^{1, *}$, Fred Martin Ralph ${ }^{2}$, Tapash Das ${ }^{3}$, Paul J. Neiman ${ }^{2}$ and}

Daniel R. Cayan ${ }^{1}$

1 U.S. Geological Survey, Scripps Institution of Oceanography, La Jolla, CA 92093, USA; E-Mail: dcayan@ucsd.edu

2 NOAA Earth Systems Research Laboratory, Boulder, CO 80305, USA; E-Mails: marty.ralph@noaa.gov (F.M.R); paul.j.neiman@noaa.gov (P.J.N);

3 Scripps Institution of Oceanography, La Jolla, CA 92093, USA; E-Mail: tadas@ucsd.edu

* Author to whom correspondence should be addressed; E-Mail: mddettin@usgs.gov; Tel.: +1-858-822-1507; Fax: +1-858-822-2028.

Received: 12 February 2011; in revised form: 3 March 2011 / Accepted: 21 March 2011 / Published: 24 March 2011

\begin{abstract}
California's highly variable climate and growing water demands combine to pose both water-supply and flood-hazard challenges to resource managers. Recently important efforts to more fully integrate the management of floods and water resources have begun, with the aim of benefitting both sectors. California is shown here to experience unusually large variations in annual precipitation and streamflow totals relative to the rest of the US, variations which mostly reflect the unusually small average number of wet days per year needed to accumulate most of its annual precipitation totals (ranging from 5 to 15 days in California). Thus whether just a few large storms arrive or fail to arrive in California can be the difference between a banner year and a drought. Furthermore California receives some of the largest 3-day storm totals in the country, rivaling in this regard the hurricane belt of the southeastern US. California's largest storms are generally fueled by landfalling atmospheric rivers (ARs). The fractions of precipitation and streamflow totals at stations across the US that are associated with ARs are documented here and, in California, contribute $20-50 \%$ of the state's precipitation and streamflow. Prospects for long-lead forecasts of these fractions are presented. From a meteorological perspective, California's water resources and floods are shown to derive from the same storms to an extent that makes integrated flood and water resources management all the more important.
\end{abstract}


Keywords: precipitation; floods; water supply; California; atmospheric rivers

\section{Introduction}

California's water resources are finite and increasingly demands and diversions are approaching their limits. The State has a Mediterranean climate with wet winters and long, warm, dry summers; so that seasonal and longer term water scarcities have been a problem throughout the State's development. On those longer times scales, California's climate, and in particular its precipitation and streamflow, are notably variable from year to year. Thus it is common wisdom that California's lot is either flood or drought, with seemingly few occasions when average conditions prevail.

Meanwhile, the State's population and economy are growing rapidly, with a current population of over 30 million people expected to grow to over 50 million by midcentury [1]. Attending this population growth will likely be significant increases in demands for water. These demands are aggravated by the fact that, while $2 / 3$ of the precipitation and runoff in California occurs in the northern third of California, roughly $3 / 4$ of the population and water demands are in the southern two-thirds of the State. In order to meet these demands, the State and Federal governments have constructed massive storage and conveyance systems that span the State.

In this challenging context, local, State and Federal agencies are innovating to balance supplies, water rights, environmental-flow requirements, and conveyance capacities to meet the growing demands. In its feast or famine way, California has also regularly been battered by major winter storms and floods that damage property on the order of billions of dollars and threaten lives; consequently the State has an even older (than most of its water supply systems) and equally extensive flood control infrastructure [2]. Increasingly in the past decade, the State has recognized that one potentially important response to growing water demands is greater coordination of flood management with water-resources management in many settings [1]. Improved floodplain management provides benefits to both flood management and the kinds of ecosystems restoration and sustainability that are increasingly required to maintain water supplies and quality. Water released from reservoirs to moderate or mitigate flood damages is water lost from many of the State's water supply systems, so that coordinating flood and supply management may increase water supplies with relatively little impact on flood-control benefits. Risks of flood damages can also be reduced by land-development and land-use practices that accommodated and avoided flood-prone areas in ways that could result in future reductions in required flood-control releases from reservoirs.

Although meteorological understanding of the midlatitude cyclones that make landfall on the west coast has a long history (e.g., [3]), and the pineapple express (PE) concept is well known by meteorologists and the public [4,5], scientific and technological advances have only recently documented just how strong the storms can be, and how they become so strong. The enlightenment has come with the recognition of the significance of a key feature within these storms that has only recently become observable with satellite technology, a feature termed the "atmospheric river" (AR; e.g., [6-10]). AR storms that impact California are a result of low-level jets along the pre-cold frontal edge of the warm sectors of major winter cyclones over the eastern North Pacific. Figure 1 
illustrates several depictions of a classic example of one of these storms, from New Years 1997 when a major PE storm drawing water vapor and warm air through an AR extending from the tropics near Hawaii to central California resulted in heavy precipitation and major flooding in California [11]. The low-level jet (often $>20 \mathrm{~m} / \mathrm{s}$ [9]) in ARs typically carries substantial water vapor, such that vertically integrated water-vapor contents total $>3 \mathrm{~cm}$ on average, as in Figure 1a, but occasionally range up to $5.5 \mathrm{~cm}$ at the extreme [8]. In combination, the winds and vapor contents yield large vapor transport rates (e.g., $>500 \mathrm{~kg}$ of water vapor per second across each meter of horizontal width of the AR as in Figure 1c. This vapor transport is concentrated into narrow and intense corridors 2,000 and more kilometers long, a few hundreds of kilometers wide, in the lowest $\sim 2.5 \mathrm{~km}$ of the atmosphere [7,9]. The atmospheric profiles within these corridors tend to be moist-statically neutral throughout the first three kilometers above the surface, so that once they encounter the slopes of California's Coastal Range and Sierra Nevada, orographic uplift is strong and orographic precipitation is copious (e.g., [10]).

Figure 1. Visualizations of atmospheric-river conditions impacting California on 1-2 January 1997: (a) SSM/I composite satellite image of integrated water vapor (cm) on 2 January modified from figure 6 in [11]; grey diamonds represent areas not overflown by SSM/I instrument that day, (b) infrared weather-satellite imagery of the Pacific Ocean basin (GOES-West) (light colors are cloud bands, coasts indicated in green), and (c) daily-average vertically integrated water-vapor transport directions and relative rates based on NCEP-NCAR Reanalysis fields [12] describing 6-hourly historical atmospheric conditions; arrow at bottom indicates length of a $1,000 \mathrm{~kg} / \mathrm{m} / \mathrm{s}$ vapor-transport vector.
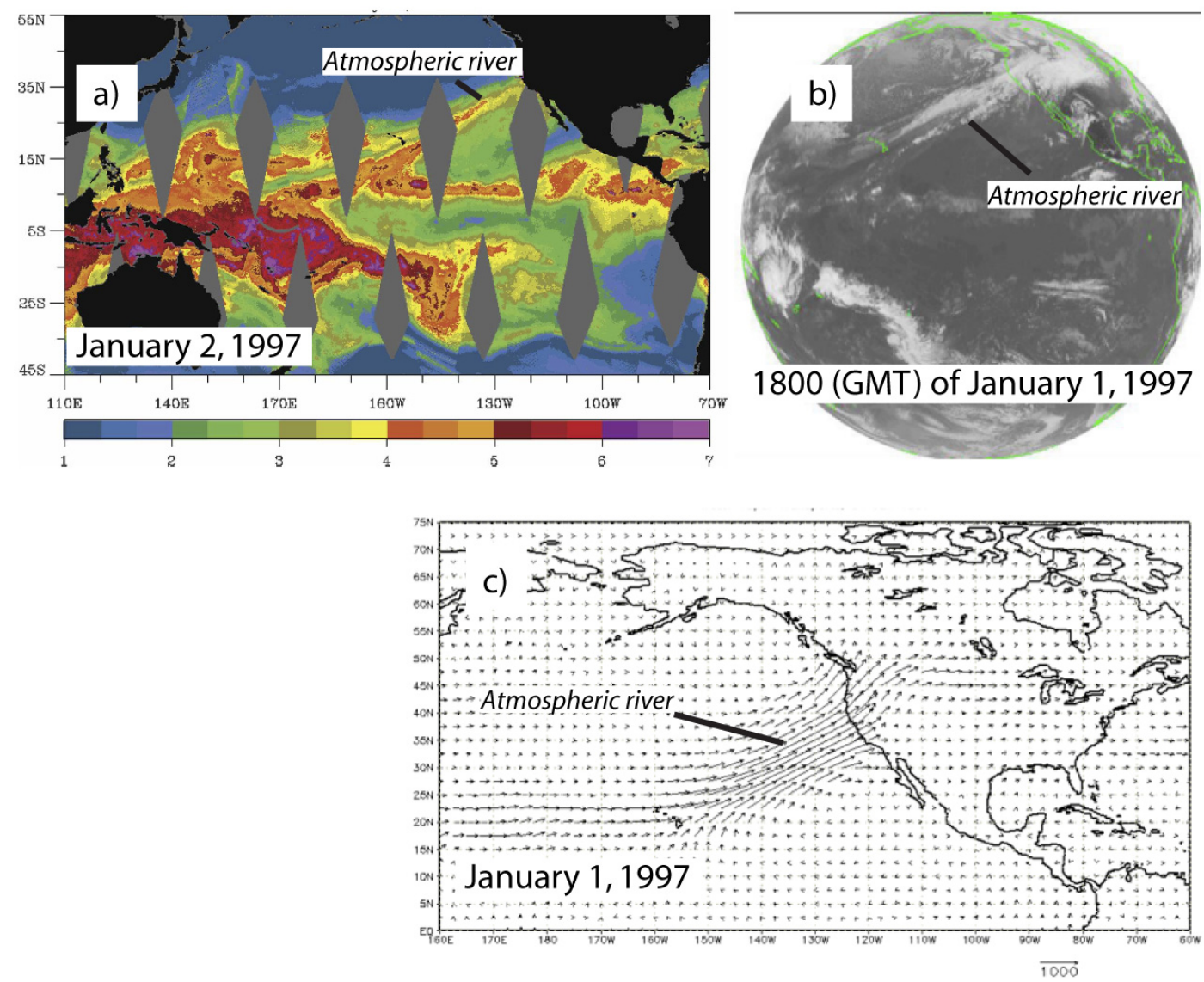
This article investigates some meteorological aspects of the connection between floods and water resources in California, with a particular focus on the dual role that AR storms play in bringing California both (1) its most dangerous storms and floods and (2) the basis for much of the State's water resources. While a series of recent papers has explored the relation between ARs and extreme rainfall and flooding in a few locations or events, as referenced above, this paper expands on these by assessing linkages to runoff and water supply across the entire western US, aided by long-term streamflow observations and a state-of-the-art hydrologic model. The paper also addresses a common question in the region: How are the AR and PE concepts related? By analyzing historical precipitation and streamflow records and simulations, along with two chronologies of atmospheric rivers making landfalls on the West Coast of the US, we will weigh and compare these two roles that ARs play in California meteorology.

\section{Data, Models and Storm Chronologies}

\subsection{Data}

Station precipitation totals analyzed here are based on daily accumulated precipitation measurements reported in the Summary of the Day (SOD; [13] and updates thereto) observations from cooperative weather stations across the United States, obtained from the National Climatic Data Center. Daily data from 1950-2008, and subsets thereof, were considered from 5,877 stations across the contiguous US, or an average of one cooperative station per 1,375 $\mathrm{km}^{2}$. Missing data were excluded, as were accumulations from multiple days reported as a multi-day totals. It should be noted that the periods of record and observation times of day differ among sites. The average length of record for the sites used is 53 years. Sites with less than 8 years of precipitation data from water years (October-September) 1998-2008 were excluded from the composites based on the AR chronology (described below); sites with less than 30 years of data from water years 1951-2008 were likewise excluded from composites based on the longer PE chronology. For the present analysis, data from other networks (e.g., snow data) were not included in our analysis to avoid network-to-network inconsistencies.

The streamflow records analyzed here are from the U.S. Geological Survey Hydro- Climatic Data Network (HCDN; [14] and updates thereto). The HCDN dataset contains daily streamflow observations from streamflow gauges that are considered to be relatively unaffected by anthropogenic influences, land-use changes, measurement changes, and measurement errors. Gauges with less than 8 years of discharge data from water years 1998-2008 were excluded from the composites based on the AR chronology (described below); gauges with less than 30 years of data from water years 1951-2008 were likewise excluded from composites based on the longer PE chronology.

\subsection{Models}

To provide some more geographically complete depictions of the contributions that ARs make to water resources of the western US, gridded daily precipitation fields used as historical inputs for a large-scale hydrologic model, and simulated daily runoff outputs from that model, will also be evaluated here. The hydrologic model is the Variable Infiltration Capacity (VIC) model [15], a 
macroscale land-surface hydrologic model that has been used in many process, sensitivity, forecast, and climate-change studies in recent years. VIC simulates (among other variables) daily runoff plus baseflow at grid cells on a 1/8-degree resolution over the conterminous US, although we focus here on basins in the western US. VIC is driven by daily maximum and minimum temperatures, precipitation, and wind speeds on the same grid. Temperatures and precipitation values for historical simulations were obtained from interpolated fields of daily observations [16] that derive much of their geographic structure from monthly historical PRISM climate fields [17]. These meteorological-input fields for VIC reflect daily observations at cooperative weather stations augmented by higher quality Global Historical Climatology Network stations [18], with interpolations to grid points based on local weather-elevation relations (e.g., lapse rates) derived separately for each historical month in the PRISM procedures. The primary gridded meteorological dataset [16] used is available for the period 1915 through 2003. To extend that dataset through 2008, daily gridded meteorological fields for the period 2004 through 2008 - from another dataset produced by the same group (the Surface Water Modeling Group at the University of Washington) based on a reduced network of stations-were appended to the primary set. This reduced-network dataset is available with near-real-time updates, and is produced and used operationally as part of a West-wide seasonal hydrologic forecast system [19]. Wind speeds, used in the hydrologic model, were interpolated from National Centers for Environmental Prediction (NCEP)/National Center for Atmospheric Research (NCAR) Reanalysis [12] data [16].

The VIC hydrologic model uses a tiled representation of the land surface within each model grid cell to allow for sub-grid variability in topography, infiltration, and land surface vegetation classes [20]. VIC forms and then melts snowpacks, and accounts for water storage and release from several soil layers, evapotranspiration, and interception losses. Surface runoff is simulated with an infiltration formulation based on the Xinanjiang model [21], while baseflow follows the ARNO model [15]. In this article, the gridded historical precipitation inputs and the simulated historical runoff-plus-baseflow, on and following days with landfalling ARs, are analyzed.

\subsection{Storm Chronologies}

For many of the analyses here, historical occurrences of landfalling ARs were identified from twice-daily Special Sensor Microwave Imager (SSM/I) imagery of vertically integrated water vapor in the atmosphere. An AR is identified here following the strategy and thresholds initially developed in [7] for one year, and extended in [22], such that narrow plumes of SSM/I vapor with values $>2 \mathrm{~cm}$ that were $>2,000 \mathrm{~km}$ long and $<1,000 \mathrm{~km}$ wide were defined as atmospheric rivers. Following this procedure, Neiman et al. ([22] and Table 1) constructed a chronology of all dates in the past dozen years when ARs have made landfall along the west coast of the conterminous US. This chronology provides a context for judging whether daily precipitation totals are associated with AR storms or non-AR storms. The chronology is based primarily on water-vapor imagery from SSM/I [23] carried on each of four Defense Meteorological Satellite Program polar orbiters that circled the globe every 102 minutes since late 1997. The SSM/I measurements are reliable [24], although water-vapor measurements can be degraded in heavy rain. However, because the SSM/I observations are used here solely for detecting long, narrow water-vapor plumes associated with atmospheric rivers (i.e., for 
pattern recognition) rather than for quantitative analysis, the degradation of vapor imagery due to heavy rainfall and small inter-satellite differences does not prove detrimental. Prior to the start of WY1998, fewer satellites were available and yielded comparatively poorer composite daily spatial coverage for assessing water-vapor plume characteristics. Consequently, those earlier years were not included in the SSM/I data analysis; however, since publication of [22], an additional 2 more recent years of ARs have been added to the chronology, with the updated chronology presented here as Table 1).

PE storms form a particularly challenging subset of ARs. PE storms are so called because they draw heat and vapor from the subtropics or tropics near Hawaii and in at least some cases (e.g., 1997, Figure 1) transport that heat and vapor towards the West Coast. Three ways of visualizing one such storm, the notorious New Years 1997 storm, were shown in Figure 1. Figure 1c shows the vertically integrated water-vapor transports on that day, illustrating - on the coarse Reanalysis [12] grid - the strong transport of vapor from over Hawaii to the West Coast, crossing the coast near $40^{\circ} \mathrm{N}$. By searching for days with transport patterns like this, with strong $(>500 \mathrm{~kg} / \mathrm{m} / \mathrm{s})$ average southwesterly vapor transports extending in an unbroken path back from the West Coast (anywhere between $32.5^{\circ} \mathrm{N}$ and $55^{\circ} \mathrm{N}$ ) into the subtropics between $170^{\circ} \mathrm{E}$ and $120^{\circ} \mathrm{W}$, a catalog of days with large-scale PE circulations has been developed [5] for the period from 1948 through 2008, with the new chronology presented here as Table 2. The PE chronology used in the present analysis was modified from that in [5] only in that the starting locations for backtracking the pineapple-express transports are along the west coast of North America (herein) rather than along a single longitude circle (in [5]), in order to more closely parallel the approach used in the AR chronology [22]. The PE chronology is longer than the AR chronology of [22], but less exhaustive because many of the SSM/I-identified ARs do not follow the simple path sought by this PE algorithm. The overlap period between the AR and PE chronologies covers 11 water years (1998-2008), during which 73 dates with PE conditions were detected. Comparison with the AR chronology, using the criterion that a date is considered an AR date if the pattern in the SSM/I imagery qualified in either the AM or PM satellite overpasses anywhere along the West Coast, shows that 71 of the 73 PE dates were also AR dates. The other 2 PE dates might also have involved ARs but may have been missed due to differences between the data and resolutions used for the $\mathrm{PE}$ and $\mathrm{AR}$ chronologies.

On average along the west coast of the conterminous U.S., the AR chronology used hereafter records 16 AR episodes per November-April, while the PE chronology records 6.4 PE episodes per season. Thus analyses using the PE chronology do not consider as many events per year as the AR-based analyses and instead focus on a particularly strong and narrow subset of ARs with a specific geographic orientation and position, but makes those analyses over a much longer historical period. 
Table 1a. Dates of SSM/I observations of landfalling atmospheric rivers (ARs; long, narrow plumes of integrated water vapor with core values $\geq \sim 2 \mathrm{~cm})$ during the water years $1998-2008$ that impacted the California coast $\left(32.5^{\circ} \mathrm{N}-41.0^{\circ} \mathrm{N}\right)$ on both the morning ascending SSM/I passes and the afternoon descending passes. The AR events in the November-April window considered in this paper are boldfaced.

\begin{tabular}{|c|c|c|c|c|c|c|c|c|c|c|c|}
\hline \# & WY1998 & WY1999 & WY2000 & WY2001 & WY2002 & WY2003 & WY2004 & WY2005 & WY2006 & WY2007 & WY2008 \\
\hline 1 & 19971002 & 19981108 & 19991014 & 20010328 & 20011011 & 20021107 & 20031005 & 20041009 & 20051026 & 20061103 & 20071019 \\
\hline 2 & 19971009 & 19981121 & 19991026 & 20010515 & 20011030 & 20021108 & 20031109 & 20041018 & 20051107 & 20061113 & 20071028 \\
\hline 3 & 19971029 & 19981123 & 19991028 & 20010516 & 20011116 & 20021109 & 20031129 & 20041026 & 20051108 & 20061117 & 20071029 \\
\hline 4 & 19971031 & 19990115 & 19991110 & 20010605 & 20011122 & 20021213 & 20031130 & 20041227 & 20051125 & 20061120 & 20071111 \\
\hline 5 & 19971106 & 19990207 & 19991111 & 20010625 & 20011129 & 20021214 & 20031205 & 20050109 & 20051129 & 20061212 & 20071204 \\
\hline 6 & 19971119 & 19990324 & 19991115 & 20010626 & 20011202 & 20021216 & 20031206 & 20050110 & 20051201 & 20070209 & 20080105 \\
\hline 7 & 19971125 & 19990325 & 20000116 & 20010627 & 20011229 & 20021227 & 20031207 & 20050322 & 20051202 & 20070210 & 20080110 \\
\hline 8 & 19971205 & 19990511 & 20000117 & 20010730 & 20020102 & 20030112 & 20031224 & 20050504 & 20051222 & 20070605 & 20080603 \\
\hline 9 & 19971214 & 19990802 & 20000118 & 20010925 & 20020106 & 20030113 & 20040216 & 20050508 & 20051228 & 20070610 & 20080622 \\
\hline 10 & 19980114 & 19990830 & 20000124 & 20010926 & 20020414 & 20030212 & 20040217 & 20050515 & 20051230 & 20070628 & 20080703 \\
\hline 11 & 19980115 & & 20000125 & & 20020529 & 20030310 & 20040527 & 20050516 & 20051231 & 20070718 & 20080730 \\
\hline 12 & 19980126 & & 20000305 & & 20020618 & 20030315 & 20040528 & 20050519 & 20060227 & & \\
\hline 13 & 19980202 & & 20000417 & & & 20030323 & 20040911 & 20050522 & 20060306 & & \\
\hline 14 & 19980203 & & 20000507 & & & 20030326 & 20040917 & 20050608 & 20060325 & & \\
\hline 15 & 19980205 & & 20000508 & & & 20030908 & & 20050609 & 20060328 & & \\
\hline 16 & 19980322 & & 20000523 & & & & & 20050617 & 20060403 & & \\
\hline 17 & 19980323 & & 20000612 & & & & & 20050709 & 20060404 & & \\
\hline 18 & 19980525 & & & & & & & 20050710 & 20060412 & & \\
\hline 19 & 19980625 & & & & & & & & 20060508 & & \\
\hline 20 & & & & & & & & & 20060519 & & \\
\hline 21 & & & & & & & & & 20060521 & & \\
\hline 22 & & & & & & & & & 20060531 & & \\
\hline 23 & & & & & & & & & 20060601 & & \\
\hline 24 & & & & & & & & & 20060602 & & \\
\hline 25 & & & & & & & & & 20060604 & & \\
\hline
\end{tabular}


Table 1b. Dates of SSM/I observations of landfalling atmospheric rivers (ARs; long, narrow plumes of integrated water vapor with core values $\geq \sim 2 \mathrm{~cm})$ during the water years $1998-2008$ that impacted the Oregon/Washington/British Columbia coast $\left(41.0^{\circ} \mathrm{N}-52.5^{\circ} \mathrm{N}\right)$ on both the morning ascending SSM/I passes and the afternoon descending passes. The AR events in the November-April window considered in this paper are boldfaced.

\begin{tabular}{|c|c|c|c|c|c|c|c|c|c|c|c|}
\hline \# & WY1998 & WY1999 & WY2000 & WY2001 & WY2002 & WY2003 & WY2004 & WY2005 & WY2006 & WY2007 & WY2008 \\
\hline 1 & 19971001 & 19981005 & 19991008 & 20001001 & 20011010 & 20021003 & 20031006 & 20041006 & 20051010 & 20061008 & 20071007 \\
\hline 2 & 19971015 & 19981006 & 19991013 & 20001008 & 20011104 & 20021027 & 20031016 & 20041008 & 20051013 & 20061015 & 20071009 \\
\hline 3 & 19971016 & 19981012 & 19991017 & 20001017 & 20011109 & 20021106 & 20031017 & 20041011 & 20051014 & 20061019 & 20071019 \\
\hline 4 & 19971026 & 19981017 & 19991030 & 20001022 & 20011114 & 20021112 & 20031018 & 20041012 & 20051021 & 20061026 & 20071022 \\
\hline 5 & 19971029 & 19981113 & 19991103 & 20001023 & 20011115 & 20021119 & 20031019 & 20041015 & 20051022 & 20061106 & 20071028 \\
\hline 6 & 19971030 & 19981114 & 19991106 & 20001123 & 20011119 & 20021212 & 20031020 & 20041016 & 20051025 & 20061107 & 20071107 \\
\hline 7 & 19971031 & 19981121 & 19991111 & 20010430 & 20020106 & 20030102 & 20031021 & 20041022 & 20051031 & 20061225 & 20071116 \\
\hline 8 & 19971102 & 19981125 & 19991112 & 20010606 & 20020107 & 20030126 & 20031022 & 20041102 & 20051101 & 20061226 & 20071117 \\
\hline 9 & 19971103 & 19981228 & 19991113 & 20010620 & 20020221 & 20030131 & 20031027 & 20041105 & 20051109 & 20070102 & 20071118 \\
\hline 10 & 19971105 & 19981229 & 19991114 & 20010703 & 20020413 & 20030313 & 20031129 & 20041106 & 20051110 & 20070122 & 20071203 \\
\hline 11 & 19971216 & 19990110 & 19991125 & 20010707 & 20020604 & 20030523 & 20040122 & 20041107 & 20051113 & 20070311 & 20071223 \\
\hline 12 & 19971228 & 19990114 & 19991215 & 20010709 & 20020625 & 20030524 & 20040526 & 20041108 & 20051224 & 20070312 & 20080102 \\
\hline 13 & 19980123 & 19990224 & 19991216 & 20010802 & 20020626 & 20030527 & 20040706 & 20041115 & 20051230 & 20070609 & 20080513 \\
\hline 14 & 19980322 & 19990227 & 20000521 & 20010803 & 20020627 & 20030626 & 20040712 & 20041124 & 20060117 & 20070613 & 20080518 \\
\hline 15 & 19980609 & 19990511 & 20000522 & 20010805 & 20020706 & 20030709 & 20040717 & 20041125 & 20060531 & 20070614 & 20080627 \\
\hline 16 & 19980614 & 19990531 & 20000523 & 20010821 & 20020710 & 20030711 & 20040718 & 20041208 & 20060616 & 20070702 & 20080705 \\
\hline 17 & 19980615 & 19990604 & 20000527 & 20010822 & 20020715 & 20030712 & 20040729 & 20041209 & 20060708 & 20070703 & 20080708 \\
\hline 18 & 19980624 & 19990623 & 20000607 & 20010825 & 20020716 & 20030714 & 20040803 & 20041210 & 20060709 & 20070714 & 20080720 \\
\hline 19 & 19980706 & 19990628 & 20000612 & 20010828 & 20020717 & 20030720 & 20040804 & 20041211 & 20060714 & 20070715 & 20080721 \\
\hline 20 & 19980712 & 19990629 & 20000614 & 20010830 & 20020718 & 20030818 & 20040817 & 20041217 & 20060720 & 20070717 & 20080726 \\
\hline 21 & 19980713 & 19990720 & 20000617 & 20010901 & 20020723 & 20030821 & 20040820 & 20050117 & 20060726 & 20070720 & 20080729 \\
\hline 22 & 19980714 & 19990726 & 20000630 & 20010903 & 20020725 & 20030825 & 20040821 & 20050118 & 20060805 & 20070721 & 20080801 \\
\hline 23 & 19980715 & 19990728 & 20000718 & 20010910 & 20020728 & 20030831 & 20040828 & 20050119 & 20060808 & 20070722 & 20080812 \\
\hline 24 & 19980716 & 19990729 & 20000719 & 20010912 & 20020729 & 20030903 & 20040829 & 20050122 & 20060828 & 20070723 & 20080823 \\
\hline
\end{tabular}


Table 1b. Cont.

\begin{tabular}{|c|c|c|c|c|c|c|c|c|c|c|c|}
\hline$\#$ & WY1998 & WY1999 & WY2000 & WY2001 & WY2002 & WY2003 & WY2004 & WY2005 & WY2006 & WY2007 & WY2008 \\
\hline 25 & 19980717 & 19990802 & 20000720 & 20010921 & 20020730 & 20030904 & 20040830 & 20050123 & 20060908 & 20070728 & 20080824 \\
\hline 26 & 19980723 & 19990817 & 20000727 & 20010922 & 20020808 & 20030905 & 20040831 & 20050327 & 20060911 & 20070812 & 20080828 \\
\hline 27 & 19980724 & 19990818 & 20000728 & 20010923 & 20020809 & 20030906 & 20040910 & 20050416 & 20060917 & 20070813 & 20080829 \\
\hline 28 & 19980809 & 19990819 & 20000729 & & 20020822 & 20030911 & 20040911 & 20050514 & 20060918 & 20070825 & 20080905 \\
\hline 29 & 19980812 & 19990821 & 20000730 & & 20020823 & 20030914 & 20040915 & 20050515 & & 20070828 & \\
\hline 30 & 19980813 & 19990822 & 20000731 & & 20020828 & 20030918 & 20040922 & 20050629 & & 20070829 & \\
\hline 31 & 19980827 & 19990823 & 20000817 & & 20020829 & 20030922 & 20040925 & 20050704 & & 20070903 & \\
\hline 32 & 19980830 & 19990824 & 20000818 & & 20020901 & 20030923 & & 20050705 & & 20070930 & \\
\hline 33 & 19980901 & 19990825 & 20000823 & & 20020902 & 20030925 & & 20050708 & & & \\
\hline 34 & 19980902 & 19990826 & 20000824 & & 20020910 & & & 20050716 & & & \\
\hline 35 & 19980903 & 19990827 & 20000825 & & 20020911 & & & 20050727 & & & \\
\hline 36 & 19980907 & 19990828 & 20000829 & & 20020912 & & & 20050728 & & & \\
\hline 37 & 19980908 & 19990829 & 20000907 & & 20020916 & & & 20050730 & & & \\
\hline 38 & 19980911 & 19990904 & 20000910 & & & & & 20050731 & & & \\
\hline 39 & 19980913 & 19990905 & 20000917 & & & & & 20050801 & & & \\
\hline 40 & 19980924 & 19990923 & 20000918 & & & & & 20050805 & & & \\
\hline 41 & & 19990929 & 20000920 & & & & & 20050817 & & & \\
\hline 42 & & & 20000929 & & & & & 20050820 & & & \\
\hline 43 & & & 20000930 & & & & & 20050821 & & & \\
\hline 44 & & & & & & & & 20050822 & & & \\
\hline 45 & & & & & & & & 20050826 & & & \\
\hline 46 & & & & & & & & 20050827 & & & \\
\hline 47 & & & & & & & & 20050908 & & & \\
\hline 48 & & & & & & & & 20050929 & & & \\
\hline 49 & & & & & & & & 20050930 & & & \\
\hline
\end{tabular}


Table 2. Dates and latitudes of landfalling pineapple-express (PE) transports of water vapor $\left(>500 \mathrm{~kg} \mathrm{~m}^{-1} \mathrm{sec}^{-1}\right)$ during the water years $1948-2008$ that impacted the California coast $\left(32.5^{\circ} \mathrm{N}-52.5^{\circ} \mathrm{N}\right)$ in NCAR/NCEP Reanalysis atmospheric fields [5,12].

\begin{tabular}{|c|c|c|c|c|c|c|c|c|c|c|c|}
\hline \multicolumn{2}{|c|}{ WY1948 } & \multicolumn{2}{|c|}{ WY1949 } & \multicolumn{2}{|c|}{ WY1950 } & \multicolumn{2}{|c|}{ WY1951 } & \multicolumn{2}{|c|}{ WY1952 } & \multicolumn{2}{|c|}{ WY1953 } \\
\hline Date & $\begin{array}{l}\text { Landfall } \\
\text { Latitude }\end{array}$ & Date & $\begin{array}{l}\text { Landfall } \\
\text { Latitude }\end{array}$ & Date & $\begin{array}{l}\text { Landfall } \\
\text { Latitude } \\
\end{array}$ & Date & $\begin{array}{l}\text { Landfall } \\
\text { Latitude }\end{array}$ & Date & $\begin{array}{l}\text { Landfall } \\
\text { Latitude }\end{array}$ & Date & $\begin{array}{l}\text { Landfall } \\
\text { Latitude }\end{array}$ \\
\hline \multirow[t]{9}{*}{19480120} & $52.5^{\circ} \mathrm{N}$ & 19490116 & $52.5^{\circ} \mathrm{N}$ & 19491029 & $52.5^{\circ} \mathrm{N}$ & 19501101 & $40.0^{\circ} \mathrm{N}$ & 19511228 & $35.0^{\circ} \mathrm{N}$ & 19521212 & $50.0^{\circ} \mathrm{N}$ \\
\hline & & & & 19491123 & $52.5^{\circ} \mathrm{N}$ & 19501117 & $35.0^{\circ} \mathrm{N}$ & 19520123 & $50.0^{\circ} \mathrm{N}$ & 19530101 & $40.0^{\circ} \mathrm{N}$ \\
\hline & & & & & & 19501120 & $37.5^{\circ} \mathrm{N}$ & 19520323 & $52.5^{\circ} \mathrm{N}$ & 19530108 & $42.5^{\circ} \mathrm{N}$ \\
\hline & & & & & & 19501202 & $42.5^{\circ} \mathrm{N}$ & & & 19530109 & $40.0^{\circ} \mathrm{N}$ \\
\hline & & & & & & 19501205 & $52.5^{\circ} \mathrm{N}$ & & & 19530122 & $42.5^{\circ} \mathrm{N}$ \\
\hline & & & & & & 19501206 & $42.5^{\circ} \mathrm{N}$ & & & 19530130 & $45.0^{\circ} \mathrm{N}$ \\
\hline & & & & & & 19501210 & $50.0^{\circ} \mathrm{N}$ & & & & \\
\hline & & & & & & 19510205 & $37.5^{\circ} \mathrm{N}$ & & & & \\
\hline & & & & & & 19510208 & $50.0^{\circ} \mathrm{N}$ & & & & \\
\hline \multicolumn{2}{|c|}{ WY1954 } & \multicolumn{2}{|c|}{ WY1955 } & \multicolumn{2}{|c|}{ WY1956 } & \multicolumn{2}{|c|}{ WY1957 } & \multicolumn{2}{|c|}{ WY1958 } & \multicolumn{2}{|c|}{ WY1959 } \\
\hline Date & $\begin{array}{l}\text { Landfall } \\
\text { Latitude }\end{array}$ & Date & $\begin{array}{l}\text { Landfall } \\
\text { Latitude }\end{array}$ & Date & $\begin{array}{l}\text { Landfall } \\
\text { Latitude } \\
\end{array}$ & Date & $\begin{array}{l}\text { Landfall } \\
\text { Latitude } \\
\end{array}$ & Date & $\begin{array}{l}\text { Landfall } \\
\text { Latitude } \\
\end{array}$ & Date & $\begin{array}{l}\text { Landfall } \\
\text { Latitude } \\
\end{array}$ \\
\hline 19531219 & $40.0^{\circ} \mathrm{N}$ & 19541104 & $52.5^{\circ} \mathrm{N}$ & 19551107 & $52.5^{\circ} \mathrm{N}$ & 19561102 & $52.5^{\circ} \mathrm{N}$ & 19580121 & $52.5^{\circ} \mathrm{N}$ & 19581201 & $45.0^{\circ} \mathrm{N}$ \\
\hline 19540307 & $35.0^{\circ} \mathrm{N}$ & 19541113 & $50.0^{\circ} \mathrm{N}$ & 19551126 & $42.5^{\circ} \mathrm{N}$ & 19561209 & $47.5^{\circ} \mathrm{N}$ & 19580122 & $52.5^{\circ} \mathrm{N}$ & 19590115 & $45.0^{\circ} \mathrm{N}$ \\
\hline \multirow[t]{9}{*}{19540308} & $37.5^{\circ} \mathrm{N}$ & 19541120 & $52.5^{\circ} \mathrm{N}$ & 19551219 & $37.5^{\circ} \mathrm{N}$ & 19561215 & $50.0^{\circ} \mathrm{N}$ & 19580217 & $50.0^{\circ} \mathrm{N}$ & 19590116 & $45.0^{\circ} \mathrm{N}$ \\
\hline & & & & 19551220 & $45.0^{\circ} \mathrm{N}$ & 19561225 & $52.5^{\circ} \mathrm{N}$ & 19580218 & $35.0^{\circ} \mathrm{N}$ & 19590214 & $40.0^{\circ} \mathrm{N}$ \\
\hline & & & & 19551221 & $45.0^{\circ} \mathrm{N}$ & 19561226 & $52.5^{\circ} \mathrm{N}$ & 19580221 & $50.0^{\circ} \mathrm{N}$ & 19590215 & $42.5^{\circ} \mathrm{N}$ \\
\hline & & & & 19560113 & $42.5^{\circ} \mathrm{N}$ & 19570112 & $32.5^{\circ} \mathrm{N}$ & 19580320 & $40.0^{\circ} \mathrm{N}$ & 19590216 & $30.0^{\circ} \mathrm{N}$ \\
\hline & & & & 19560114 & $37.5^{\circ} \mathrm{N}$ & 19570222 & $42.5^{\circ} \mathrm{N}$ & 19580321 & $30.0^{\circ} \mathrm{N}$ & & \\
\hline & & & & 19560118 & $52.5^{\circ} \mathrm{N}$ & 19570224 & $40.0^{\circ} \mathrm{N}$ & & & & \\
\hline & & & & 19560119 & $40.0^{\circ} \mathrm{N}$ & 19570225 & $42.5^{\circ} \mathrm{N}$ & & & & \\
\hline & & & & 19560120 & $52.5^{\circ} \mathrm{N}$ & 19570226 & $40.0^{\circ} \mathrm{N}$ & & & & \\
\hline & & & & & & 19570610 & $52.5^{\circ} \mathrm{N}$ & & & & \\
\hline
\end{tabular}


Table 2. Cont.

\begin{tabular}{|c|c|c|c|c|c|c|c|c|c|c|c|}
\hline \multicolumn{2}{|c|}{ WY1960 } & \multicolumn{2}{|c|}{ WY1961 } & \multicolumn{2}{|c|}{ WY1962 } & \multicolumn{2}{|c|}{ WY1963 } & \multicolumn{2}{|c|}{ WY1964 } & \multicolumn{2}{|c|}{ WY1965 } \\
\hline Date & $\begin{array}{l}\text { Landfall } \\
\text { Latitude }\end{array}$ & Date & $\begin{array}{l}\text { Landfall } \\
\text { Latitude } \\
\end{array}$ & Date & $\begin{array}{l}\text { Landfall } \\
\text { Latitude } \\
\end{array}$ & Date & $\begin{array}{l}\text { Landfall } \\
\text { Latitude } \\
\end{array}$ & Date & $\begin{array}{l}\text { Landfall } \\
\text { Latitude } \\
\end{array}$ & Date & $\begin{array}{l}\text { Landfall } \\
\text { Latitude } \\
\end{array}$ \\
\hline 19591122 & $45.0^{\circ} \mathrm{N}$ & 19601212 & $47.5^{\circ} \mathrm{N}$ & 19611013 & $52.5^{\circ} \mathrm{N}$ & 19621027 & $52.5^{\circ} \mathrm{N}$ & 19631124 & $52.5^{\circ} \mathrm{N}$ & 19641221 & $42.5^{\circ} \mathrm{N}$ \\
\hline 19600128 & $42.5^{\circ} \mathrm{N}$ & 19610110 & $47.5^{\circ} \mathrm{N}$ & 19611125 & $32.5^{\circ} \mathrm{N}$ & 19630130 & $37.5^{\circ} \mathrm{N}$ & 19631125 & $45.0^{\circ} \mathrm{N}$ & 19641222 & $35.0^{\circ} \mathrm{N}$ \\
\hline 19600129 & $45.0^{\circ} \mathrm{N}$ & 19610115 & $50.0^{\circ} \mathrm{N}$ & 19620104 & $52.5^{\circ} \mathrm{N}$ & 19630131 & $37.5^{\circ} \mathrm{N}$ & 19631222 & $52.5^{\circ} \mathrm{N}$ & 19650112 & $52.5^{\circ} \mathrm{N}$ \\
\hline 19600205 & $50.0^{\circ} \mathrm{N}$ & 19610201 & $47.5^{\circ} \mathrm{N}$ & 19620107 & $52.5^{\circ} \mathrm{N}$ & 19630201 & $35.0^{\circ} \mathrm{N}$ & 19631224 & $47.5^{\circ} \mathrm{N}$ & & \\
\hline \multirow{4}{*}{19600206} & & 19610205 & $47.5^{\circ} \mathrm{N}$ & & & 19630203 & $47.5^{\circ} \mathrm{N}$ & & & & \\
\hline & & & & & & 19630204 & $50.0^{\circ} \mathrm{N}$ & & & & \\
\hline & & & & & & 19630206 & $50.0^{\circ} \mathrm{N}$ & & & & \\
\hline & & & & & & 19630327 & $35.0^{\circ} \mathrm{N}$ & & & & \\
\hline \multicolumn{2}{|c|}{ WY1966 } & \multicolumn{2}{|c|}{ WY1967 } & \multicolumn{2}{|c|}{ WY1968 } & \multicolumn{2}{|c|}{ WY1969 } & \multicolumn{2}{|c|}{ WY1970 } & \multicolumn{2}{|c|}{ WY1971 } \\
\hline Date & $\begin{array}{l}\text { Landfall } \\
\text { Latitude }\end{array}$ & Date & $\begin{array}{l}\text { Landfall } \\
\text { Latitude } \\
\end{array}$ & Date & $\begin{array}{l}\text { Landfall } \\
\text { Latitude } \\
\end{array}$ & Date & $\begin{array}{l}\text { Landfall } \\
\text { Latitude } \\
\end{array}$ & Date & $\begin{array}{l}\text { Landfall } \\
\text { Latitude } \\
\end{array}$ & Date & $\begin{array}{l}\text { Landfall } \\
\text { Latitude } \\
\end{array}$ \\
\hline 19651112 & $42.5^{\circ} \mathrm{N}$ & 19670126 & $40.0^{\circ} \mathrm{N}$ & 19680114 & $40.0^{\circ} \mathrm{N}$ & 19681108 & $45.0^{\circ} \mathrm{N}$ & 19700108 & $37.5^{\circ} \mathrm{N}$ & 19701123 & $42.5^{\circ} \mathrm{N}$ \\
\hline 19651115 & $32.5^{\circ} \mathrm{N}$ & 19670202 & $47.5^{\circ} \mathrm{N}$ & 19680117 & $50.0^{\circ} \mathrm{N}$ & 19690103 & $52.5^{\circ} \mathrm{N}$ & 19700113 & $45.0^{\circ} \mathrm{N}$ & 19701124 & $42.5^{\circ} \mathrm{N}$ \\
\hline 19651122 & $30.0^{\circ} \mathrm{N}$ & 19670208 & $52.5^{\circ} \mathrm{N}$ & 19680118 & $47.5^{\circ} \mathrm{N}$ & 19690104 & $45.0^{\circ} \mathrm{N}$ & 19700114 & $35.0^{\circ} \mathrm{N}$ & 19710115 & $42.5^{\circ} \mathrm{N}$ \\
\hline 19651123 & $32.5^{\circ} \mathrm{N}$ & 19630316 & $35.0) \mathrm{N}$ & 19680119 & $47.5^{\circ} \mathrm{N}$ & 19690111 & $35.0^{\circ} \mathrm{N}$ & 19700115 & $40.0^{\circ} \mathrm{N}$ & 19710116 & $47.5^{\circ} \mathrm{N}$ \\
\hline \multirow[t]{7}{*}{19660329} & $52.5^{\circ} \mathrm{N}$ & & & 19680120 & $50.0^{\circ} \mathrm{N}$ & 19690112 & $35.0^{\circ} \mathrm{N}$ & 19700117 & $37.5^{\circ} \mathrm{N}$ & 19710117 & $45.0^{\circ} \mathrm{N}$ \\
\hline & & & & 19680121 & $50.0^{\circ} \mathrm{N}$ & 19690113 & $32.5^{\circ} \mathrm{N}$ & 19700118 & $47.5^{\circ} \mathrm{N}$ & 19710118 & $42.5^{\circ} \mathrm{N}$ \\
\hline & & & & 19680122 & $52.5^{\circ} \mathrm{N}$ & 19690118 & $35.0^{\circ} \mathrm{N}$ & 19700121 & $45.0^{\circ} \mathrm{N}$ & 19710129 & $52.5^{\circ} \mathrm{N}$ \\
\hline & & & & 19680217 & $42.5^{\circ} \mathrm{N}$ & 19690124 & $35.0^{\circ} \mathrm{N}$ & 19700122 & $45.0^{\circ} \mathrm{N}$ & 19710130 & $50.0^{\circ} \mathrm{N}$ \\
\hline & & & & 19680218 & $40.0^{\circ} \mathrm{N}$ & 19690125 & $32.5^{\circ} \mathrm{N}$ & 19700215 & $42.5^{\circ} \mathrm{N}$ & 19710322 & $40.0^{\circ} \mathrm{N}$ \\
\hline & & & & 19680220 & $40.0^{\circ} \mathrm{N}$ & 19690601 & $52.5^{\circ} \mathrm{N}$ & & & & \\
\hline & & & & 19680221 & $42.5^{\circ} \mathrm{N}$ & & & & & & \\
\hline
\end{tabular}


Table 2. Cont.

\begin{tabular}{|c|c|c|c|c|c|c|c|c|c|c|c|}
\hline \multicolumn{2}{|c|}{ WY1972 } & \multicolumn{2}{|c|}{ WY1973 } & \multicolumn{2}{|c|}{ WY1974 } & \multicolumn{2}{|c|}{ WY1975 } & \multicolumn{2}{|c|}{ WY1976 } & \multicolumn{2}{|c|}{ WY1977 } \\
\hline Date & $\begin{array}{l}\text { Landfall } \\
\text { Latitude }\end{array}$ & Date & $\begin{array}{l}\text { Landfall } \\
\text { Latitude }\end{array}$ & Date & $\begin{array}{l}\text { Landfall } \\
\text { Latitude }\end{array}$ & Date & $\begin{array}{l}\text { Landfall } \\
\text { Latitude }\end{array}$ & Date & $\begin{array}{l}\text { Landfall } \\
\text { Latitude }\end{array}$ & Date & $\begin{array}{l}\text { Landfall } \\
\text { Latitude }\end{array}$ \\
\hline 19720309 & $50.0^{\circ} \mathrm{N}$ & 19721031 & $50.0^{\circ} \mathrm{N}$ & 19731108 & $40.0^{\circ} \mathrm{N}$ & 19750117 & $47.5^{\circ} \mathrm{N}$ & 19751223 & $45.0^{\circ} \mathrm{N}$ & 19770116 & $52.5^{\circ} \mathrm{N}$ \\
\hline \multirow[t]{6}{*}{19720314} & $52.5^{\circ} \mathrm{N}$ & 19721216 & $42.5^{\circ} \mathrm{N}$ & 19731111 & $37.5^{\circ} \mathrm{N}$ & & & 19751225 & $50.0^{\circ} \mathrm{N}$ & 19770117 & $52.5^{\circ} \mathrm{N}$ \\
\hline & & 19721219 & $40.0^{\circ} \mathrm{N}$ & 19731215 & $45.0^{\circ} \mathrm{N}$ & & & 19751227 & $52.5^{\circ} \mathrm{N}$ & 19770210 & $47.5^{\circ} \mathrm{N}$ \\
\hline & & 19721220 & $42.5^{\circ} \mathrm{N}$ & 19740114 & $45.0^{\circ} \mathrm{N}$ & & & 19760126 & $52.5^{\circ} \mathrm{N}$ & & \\
\hline & & & & 19740116 & $37.5^{\circ} \mathrm{N}$ & & & & & & \\
\hline & & & & 19740117 & $35.0^{\circ} \mathrm{N}$ & & & & & & \\
\hline & & & & 19740314 & $40.0^{\circ} \mathrm{N}$ & & & & & & \\
\hline \multicolumn{2}{|c|}{ WY1978 } & \multicolumn{2}{|c|}{ WY1979 } & \multicolumn{2}{|c|}{ WY1980 } & \multicolumn{2}{|c|}{ WY1981 } & \multicolumn{2}{|c|}{ WY1982 } & \multicolumn{2}{|c|}{ WY1983 } \\
\hline Date & $\begin{array}{l}\text { Landfall } \\
\text { Latitude }\end{array}$ & Date & $\begin{array}{l}\text { Landfall } \\
\text { Latitude }\end{array}$ & Date & $\begin{array}{l}\text { Landfall } \\
\text { Latitude }\end{array}$ & Date & $\begin{array}{l}\text { Landfall } \\
\text { Latitude }\end{array}$ & Date & $\begin{array}{l}\text { Landfall } \\
\text { Latitude }\end{array}$ & Date & $\begin{array}{l}\text { Landfall } \\
\text { Latitude }\end{array}$ \\
\hline 19771124 & $42.5^{\circ} \mathrm{N}$ & 19790109 & $40.0^{\circ} \mathrm{N}$ & 19791217 & $45.0^{\circ} \mathrm{N}$ & 19801210 & $52.5^{\circ} \mathrm{N}$ & 19820107 & $50.0^{\circ} \mathrm{N}$ & 19830102 & $42.5^{\circ} \mathrm{N}$ \\
\hline 19771125 & $47.5^{\circ} \mathrm{N}$ & 19790110 & $40.0^{\circ} \mathrm{N}$ & 19800111 & $32.5^{\circ} \mathrm{N}$ & 19801224 & $45.0^{\circ} \mathrm{N}$ & 19820108 & $52.5^{\circ} \mathrm{N}$ & 19830106 & $40.0^{\circ} \mathrm{N}$ \\
\hline 19771127 & $45.0^{\circ} \mathrm{N}$ & 19790212 & $40.0^{\circ} \mathrm{N}$ & 19800201 & $45.0^{\circ} \mathrm{N}$ & 19801225 & $45.0^{\circ} \mathrm{N}$ & 19820124 & $42.5^{\circ} \mathrm{N}$ & 19830125 & $42.5^{\circ} \mathrm{N}$ \\
\hline 19771226 & $32.5^{\circ} \mathrm{N}$ & 19790213 & $35.0^{\circ} \mathrm{N}$ & 19800214 & $30.0^{\circ} \mathrm{N}$ & 19810118 & $50.0^{\circ} \mathrm{N}$ & 19820212 & $47.5^{\circ} \mathrm{N}$ & 19830126 & $40.0^{\circ} \mathrm{N}$ \\
\hline 19771227 & $32.5^{\circ} \mathrm{N}$ & 19790305 & $50.0^{\circ} \mathrm{N}$ & 19800215 & $35.0^{\circ} \mathrm{N}$ & 19810119 & $50.0^{\circ} \mathrm{N}$ & 19820213 & $45.0^{\circ} \mathrm{N}$ & 19830210 & $50.0^{\circ} \mathrm{N}$ \\
\hline \multirow[t]{7}{*}{19780107} & $50.0^{\circ} \mathrm{N}$ & 19790425 & $35.0^{\circ} \mathrm{N}$ & & & 19810121 & $40.0^{\circ} \mathrm{N}$ & 19820217 & $50.0^{\circ} \mathrm{N}$ & 19830211 & $52.5^{\circ} \mathrm{N}$ \\
\hline & & & & & & 19810212 & $45.0^{\circ} \mathrm{N}$ & 19820218 & $47.5^{\circ} \mathrm{N}$ & 19830217 & $45.0^{\circ} \mathrm{N}$ \\
\hline & & & & & & 19810213 & $40.0^{\circ} \mathrm{N}$ & 19820219 & $40.0^{\circ} \mathrm{N}$ & 19820228 & $35.0^{\circ} \mathrm{N}$ \\
\hline & & & & & & 19810214 & $42.5^{\circ} \mathrm{N}$ & 19820409 & $35.0^{\circ} \mathrm{N}$ & 19830301 & $35.0^{\circ} \mathrm{N}$ \\
\hline & & & & & & 19810215 & $47.5^{\circ} \mathrm{N}$ & 19820410 & $35.0^{\circ} \mathrm{N}$ & 19830308 & $35.0^{\circ} \mathrm{N}$ \\
\hline & & & & & & 19810217 & $42.5^{\circ} \mathrm{N}$ & 19820411 & $32.5^{\circ} \mathrm{N}$ & & \\
\hline & & & & & & 19810218 & $42.5^{\circ} \mathrm{N}$ & & & & \\
\hline
\end{tabular}


Table 2. Cont.

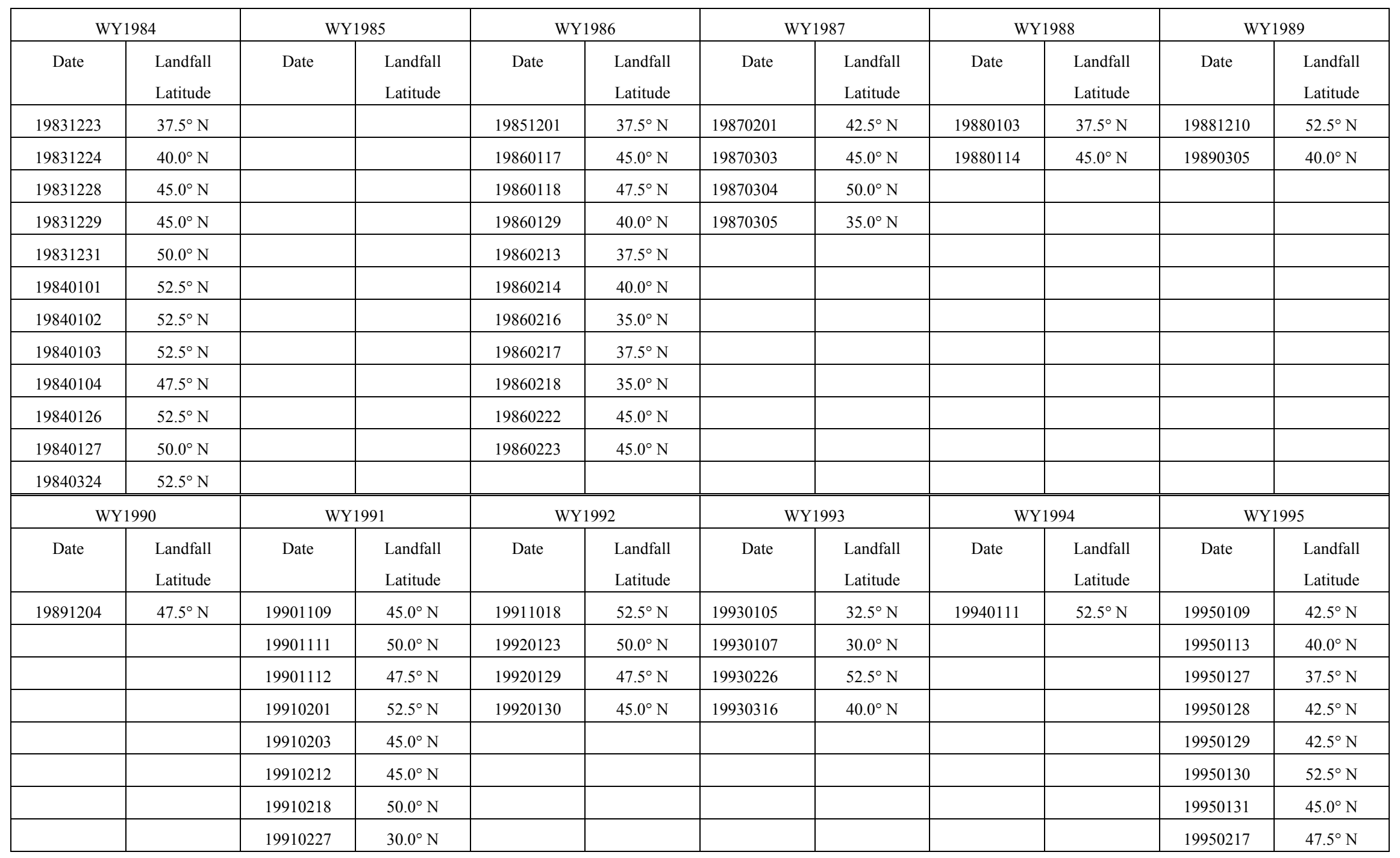


Table 2. Cont

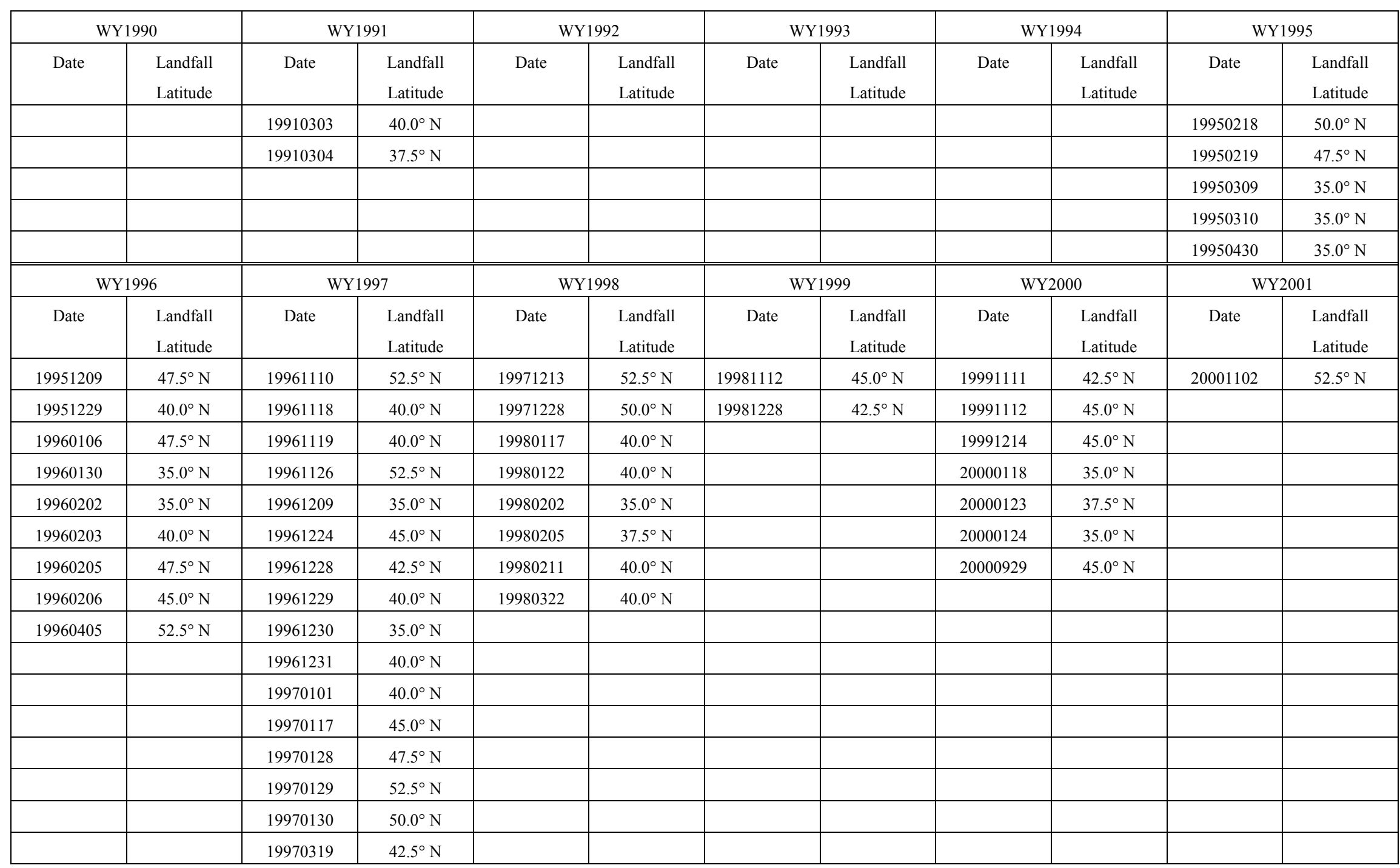


Table 2. Cont.

\begin{tabular}{|c|c|c|c|c|c|c|c|c|c|c|c|c|c|}
\hline \multicolumn{2}{|c|}{ WY2002 } & \multicolumn{2}{|c|}{ WY2003 } & \multicolumn{2}{|c|}{ WY2004 } & \multicolumn{2}{|c|}{ WY2005 } & \multicolumn{2}{|c|}{ WY2006 } & \multicolumn{2}{|c|}{ WY2007 } & \multicolumn{2}{|c|}{ WY2008 } \\
\hline Date & $\begin{array}{l}\text { Landfall } \\
\text { Latitude }\end{array}$ & Date & $\begin{array}{l}\text { Landfall } \\
\text { Latitude }\end{array}$ & Date & $\begin{array}{l}\text { Landfall } \\
\text { Latitude }\end{array}$ & Date & $\begin{array}{l}\text { Landfall } \\
\text { Latitude }\end{array}$ & Date & $\begin{array}{l}\text { Landfall } \\
\text { Latitude }\end{array}$ & Date & $\begin{array}{l}\text { Landfall } \\
\text { Latitude }\end{array}$ & Date & $\begin{array}{l}\text { Landfall } \\
\text { Latitude }\end{array}$ \\
\hline 20011114 & $47.5^{\circ} \mathrm{N}$ & 20030101 & $50.0^{\circ} \mathrm{N}$ & 20031017 & $47.5^{\circ} \mathrm{N}$ & 20041011 & $50.0^{\circ} \mathrm{N}$ & 20051219 & $42.5^{\circ} \mathrm{N}$ & 20061105 & $47.5^{\circ} \mathrm{N}$ & 20071202 & $47.5^{\circ} \mathrm{N}$ \\
\hline 20011229 & $30.0^{\circ} \mathrm{N}$ & 20030102 & $45.0^{\circ} \mathrm{N}$ & 20031020 & $45.0^{\circ} \mathrm{N}$ & 20041106 & $47.5^{\circ} \mathrm{N}$ & 20051220 & $50.0^{\circ} \mathrm{N}$ & 20061106 & $50.0^{\circ} \mathrm{N}$ & 20071203 & $45.0^{\circ} \mathrm{N}$ \\
\hline 20011231 & $40.0^{\circ} \mathrm{N}$ & 20030122 & $42.5^{\circ} \mathrm{N}$ & 20031204 & $40.0^{\circ} \mathrm{N}$ & 20041113 & $52.5^{\circ} \mathrm{N}$ & 20051223 & $47.5^{\circ} \mathrm{N}$ & 20061115 & $47.5^{\circ} \mathrm{N}$ & 20071204 & $40.0^{\circ} \mathrm{N}$ \\
\hline 20020101 & $40.0^{\circ} \mathrm{N}$ & 20030124 & $47.5^{\circ} \mathrm{N}$ & 20040105 & $45.0^{\circ} \mathrm{N}$ & 20041114 & $47.5^{\circ} \mathrm{N}$ & 20060226 & $47.5^{\circ} \mathrm{N}$ & 20061225 & $42.5^{\circ} \mathrm{N}$ & & \\
\hline 20020106 & $42.5^{\circ} \mathrm{N}$ & 20030125 & $50.0^{\circ} \mathrm{N}$ & 20040215 & $42.5^{\circ} \mathrm{N}$ & 20041209 & $40.0^{\circ} \mathrm{N}$ & 20060227 & $40.0^{\circ} \mathrm{N}$ & 20061231 & $50.0^{\circ} \mathrm{N}$ & & \\
\hline 20020107 & $42.5^{\circ} \mathrm{N}$ & 20030126 & $45.0^{\circ} \mathrm{N}$ & 20040216 & $37.5^{\circ} \mathrm{N}$ & 20041210 & $47.5^{\circ} \mathrm{N}$ & & & 20070101 & $50.0^{\circ} \mathrm{N}$ & & \\
\hline 20020409 & $37.5^{\circ} \mathrm{N}$ & 20030130 & $42.5^{\circ} \mathrm{N}$ & & & 20050116 & $50.0^{\circ} \mathrm{N}$ & & & 20070122 & $52.5^{\circ} \mathrm{N}$ & & \\
\hline \multirow[t]{7}{*}{20020412} & $42.5^{\circ} \mathrm{N}$ & & & & & 20050117 & $50.0^{\circ} \mathrm{N}$ & & & 20070214 & $52.5^{\circ} \mathrm{N}$ & & \\
\hline & & & & & & 20050118 & $47.5^{\circ} \mathrm{N}$ & & & 20070215 & $45.0^{\circ} \mathrm{N}$ & & \\
\hline & & & & & & 20050119 & $47.5^{\circ} \mathrm{N}$ & & & 20070310 & $45.0^{\circ} \mathrm{N}$ & & \\
\hline & & & & & & 20050122 & $52.5^{\circ} \mathrm{N}$ & & & 20070311 & $47.5^{\circ} \mathrm{N}$ & & \\
\hline & & & & & & 20050123 & $52.5^{\circ} \mathrm{N}$ & & & 20070505 & $52.5^{\circ} \mathrm{N}$ & & \\
\hline & & & & & & 20050305 & $50.0^{\circ} \mathrm{N}$ & & & & & & \\
\hline & & & & & & 20050326 & $47.5^{\circ} \mathrm{N}$ & & & & & & \\
\hline
\end{tabular}




\section{Precipitation Variability in California}

At the core of many of California's water issues is its generally high degree of year-to-year and within-year precipitation variability. Addressing year-to-year variability, Figures $2 \mathrm{a}-\mathrm{b}$ show coefficients of variation (standard deviation/mean) of water-year (October-September) precipitation (2a) and streamflow (2b) totals at sites across the conterminous US. Clearly precipitation and, somewhat less so, streamflow in California are proportionally more variable from year to year than are flows in other parts of the West Coast, and broadly more variable than most parts of the western and eastern states. Only some rivers contributing to the Mississippi from the west and in eastern Texas are similarly variable. The larger variations in California necessitate heroic levels of management of the State's water resources to accommodate wider swings of wet and dry years than in any other state.

Figure 2. Coefficients of variation of water-year (a) precipitation and (b) streamflow totals at long-term monitoring stations across the conterminous US, from water year 1951-2008, along with (c) tallies of the minimum number of wet days per year, on average, that provide half of the year's precipitation in the western States.

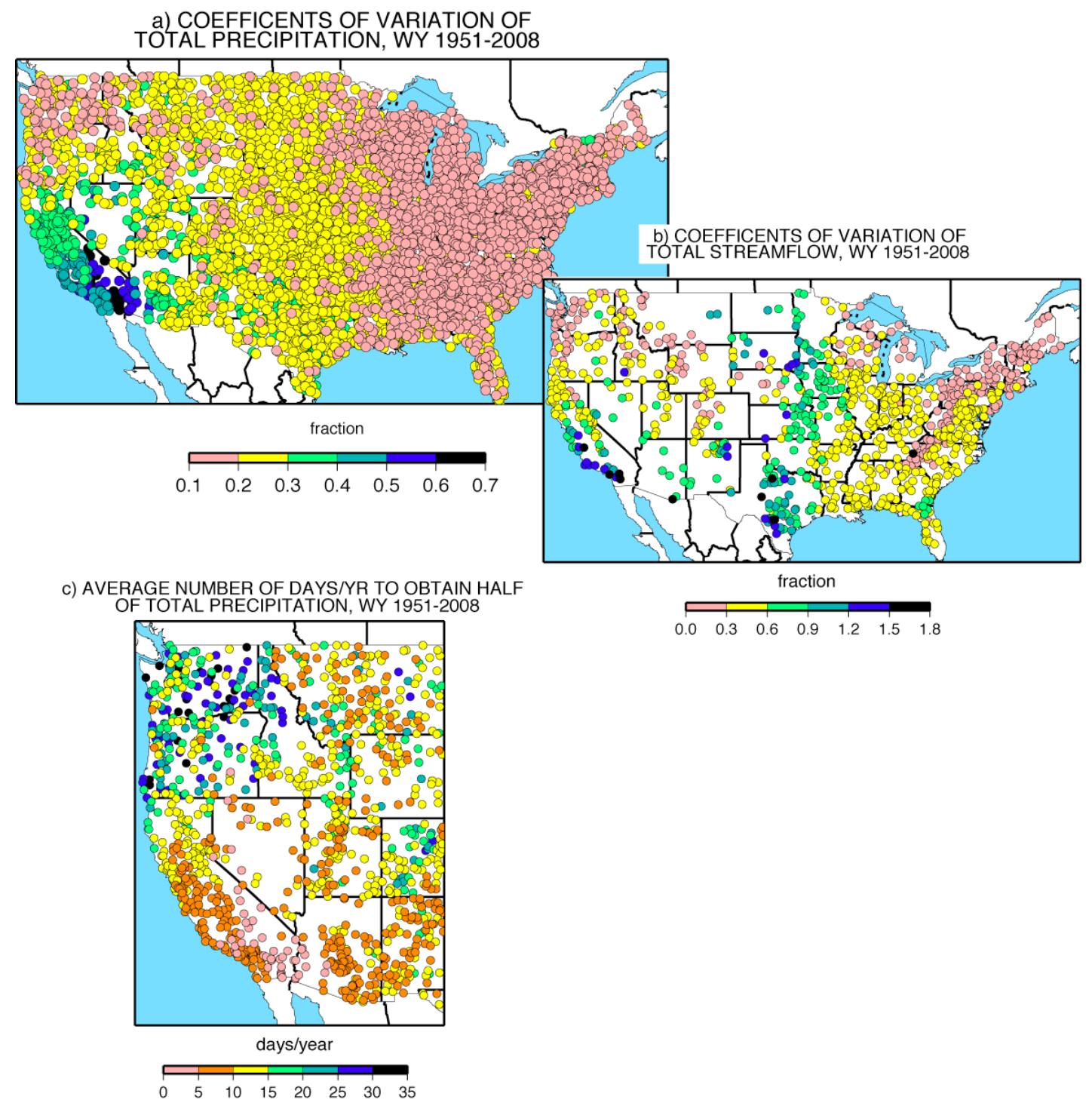


Much of the large variability of California's precipitation and water resources in turn derives from the fact that its annual precipitation totals are dependent on precipitation from a relatively few storms. If just a couple of storms do not arrive in California, or yield significantly less precipitation than needed, in a given year, that year's precipitation total and water resources suffer disproportionately, compared to other regions. Alternatively a relatively few large or "extra" storms may result in a particularly wet year. Figure $2 \mathrm{c}$ shows the average number of wet days (days with a nonzero, nontrace precipitation amount) per year required to accumulate half of the water year's precipitation total. In much of California, a third to a half of all the precipitation that falls, on average, falls in only 5 to 10 wet days per year. Furthermore, even allowing for intervening dry days, a far shorter wet season is available in California to accumulate $2 / 3$ of the total precipitation than in any other State $(<125$ days per year in California versus $>150$ to 250 in other states), as shown in Figure 3.

Figure 3. Number of days (L67) required to accumulate $67 \%$ of the annual climatological total precipitation, calculated from long-term daily mean precipitation over the period of record available at each station prior to 2000. The beginning of the L67 "season" is the day for which L67 length is at its minimum [25].

\section{a. L67: Time (days) to accumulate $67 \%$ of annual total precip Mean of length of record, daily CO-OP and 1st order stations}

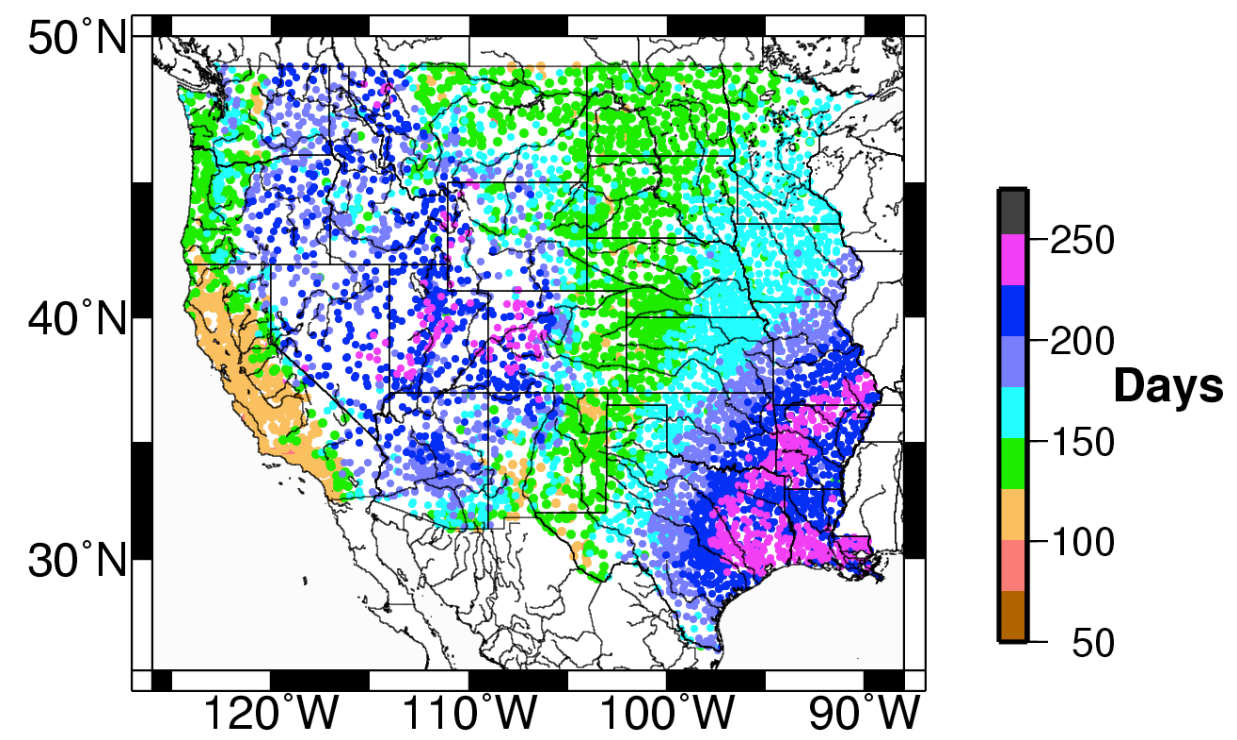

\section{AR Precipitation and Floods}

To properly understand the intensity of the (usually few) large storms that contribute so much to California's precipitation totals but that also go beyond resource to yield some of the State's greatest hazards, it is worthwhile to consider the largest recorded storm precipitation totals, nationwide [26]. Colored dots in Figure 4 show all recorded occasions since 1950 when 3-day precipitation totals have exceeded $40 \mathrm{~cm}$ at cooperative weather stations in the conterminous US. These largest storms have concentrated in two regions, along the hurricane and tropical storm belts of Texas through the Carolinas in the southeastern US and in California. The large storms in California have occurred 
primarily in winter whereas the large southeastern storms have occurred in summer [26]. The frequency of these largest storms are comparable from the southeast to California, with 0.48 reports/year/1,000 stations east of $105^{\circ} \mathrm{W}$ and 0.53 reports/year/1,000 stations west of $105^{\circ} \mathrm{W}$. Notably, at least within the past dozen years (i.e., the period with suitable SSM/I imagery), a comparison of the large storms counted in Figure 4 to the chronology of ARs making land fall in California (Table 1) indicates that all of the large California storms in Figure 4 have been associated with ARs; in the longer Reanalysis period since 1948, 69\% of the large storms in California in Figure 4 are associated with PEs. Thus extreme precipitation events, associated with landfalling ARs in California, have been historically comparable, in magnitudes and frequencies (for 3-day precipitation totals), to those mostly associated with landfalling hurricanes and tropical storms in the southeastern US and are among the largest historical storms in the conterminous US.

Figure 4. Numbers of reported occasions of (nonoverlapping) 3-day precipitation totals at cooperative weather stations that exceeded $40 \mathrm{~cm}$, from 1950-2008.

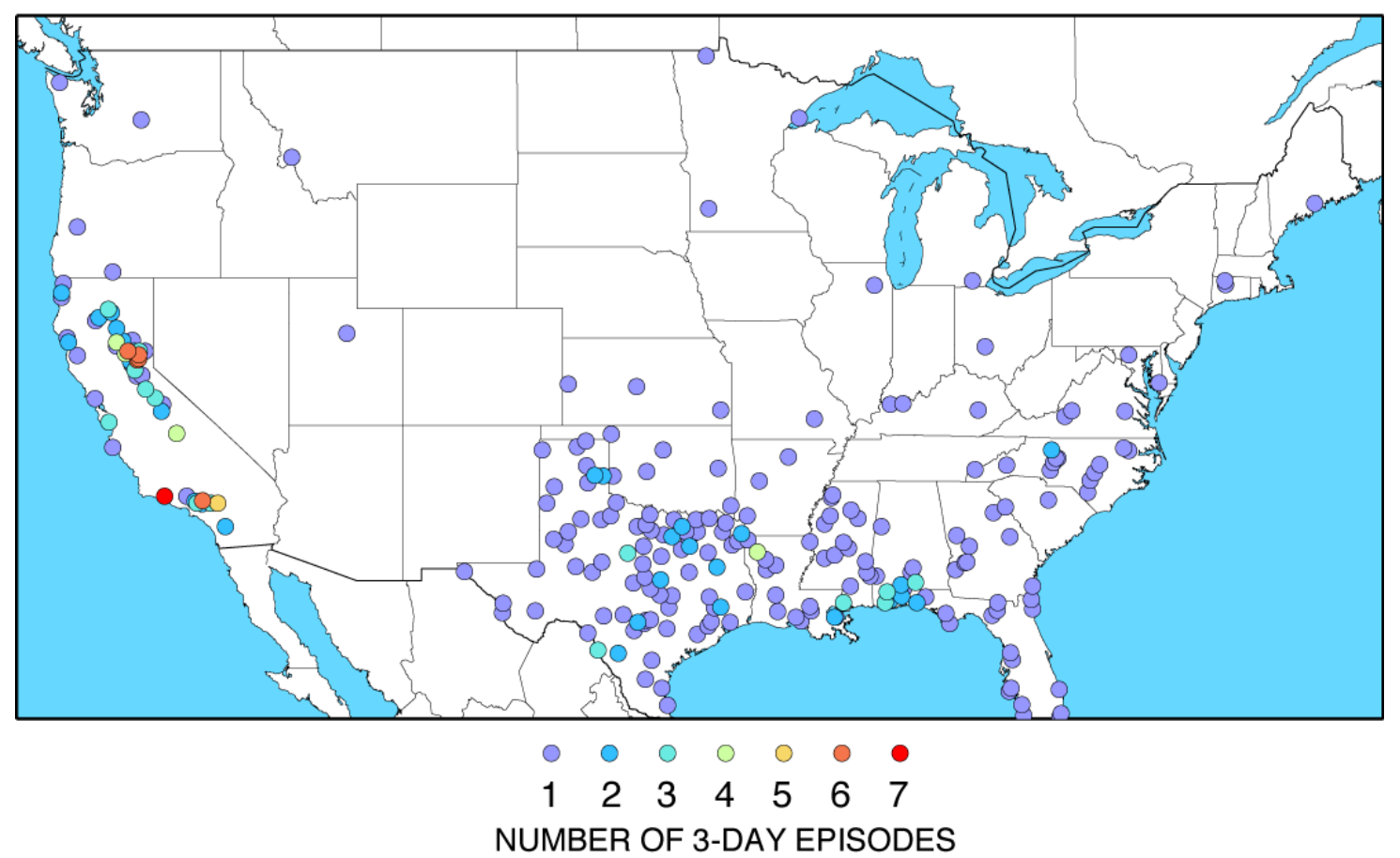

As a result of these large AR precipitation totals in California, as well as the typically warm AR-storm conditions that favor high snowlines and widespread rainfall [22], historical ARs and PEs in California (and elsewhere along the West Coast) have frequently resulted in flooding. Indeed, ARs are the primary (and, in some settings, essentially only) cause of flooding of California rivers. For example, all declared floods of the Russian River near Guerneville north of San Francisco in the period of SSM/I-based AR chronologies (water years 1998-2010) have been associated directly with ARs making landfall on the central California coast [10]. Comparison of the longer, but more restrictive, chronology of landfalling PE storms on the West Coast since 1948 to streamflows [5] has shown that streamflow increments associated with PE storms in the Merced and American Rivers of the western Sierra Nevada of Central California are an order of magnitude larger, at each recurrence level, than corresponding increments associated with non-PE storms (e.g., for the American River, Figure 5). As a 
final example, all cool-season floods since 1948 in the Carson River basin in the eastern, rain-shadowed part of the Sierra Nevada have been associated with PEs [27]. Farther north, in Washington and Oregon, AR and PE storms produce roughly twice as much precipitation as the overall average storms, and have been associated with several recent (at least) extreme flooding events $[8,22]$.

Figure 5. Exceedence probabilities for day-to-day changes in river discharge of the North Fork American River above North Fork Dam, above Sacramento, CA, under various December-February storm and precipitation conditions.

DECEMBER-FEBRUARY DAILY DISCHARGE-CHANGE DISTRIBUTIONS

North Fork American River, 1949-1999

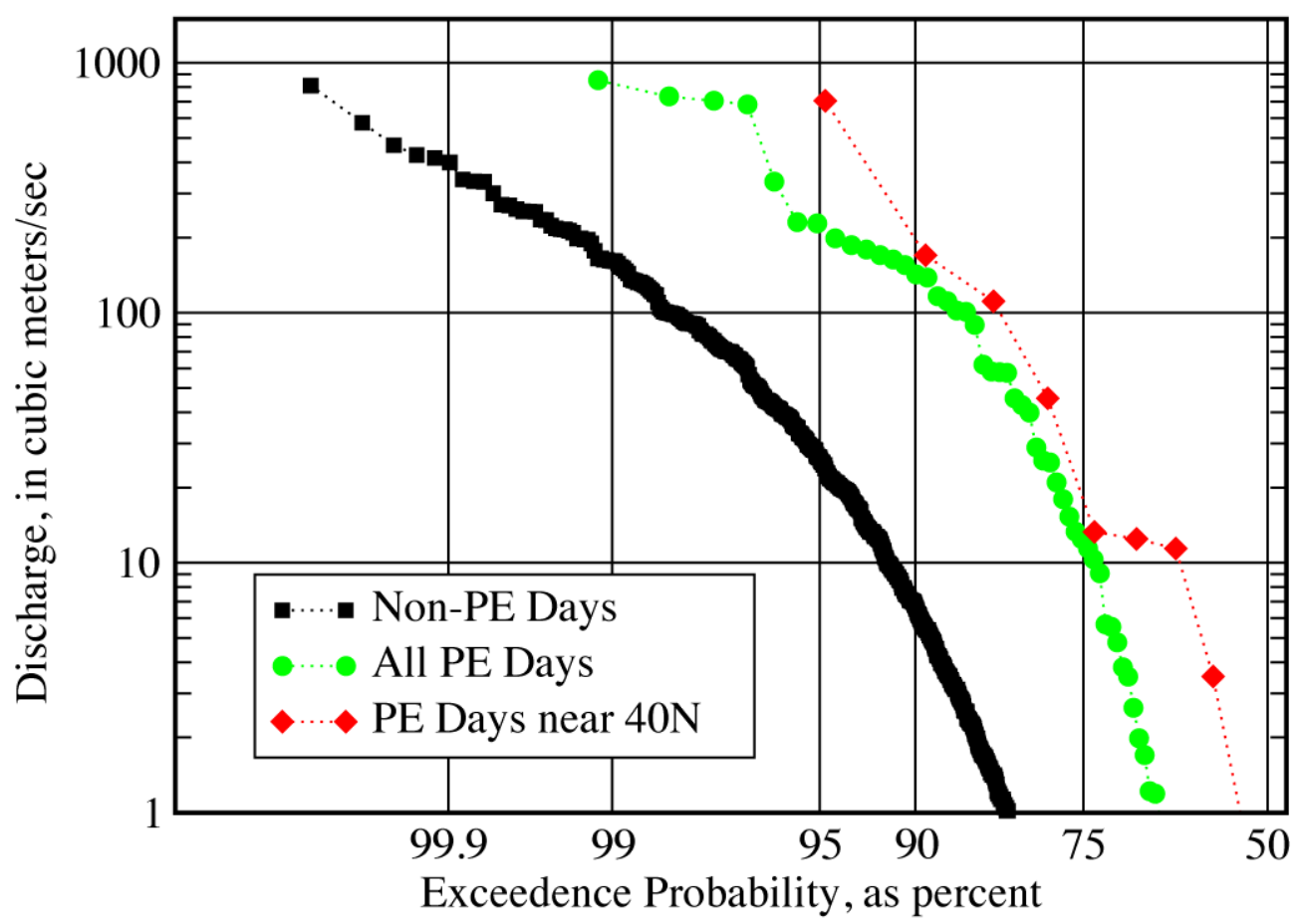

\section{Contributions of ARs to Overall Precipitation and Streamflow}

\subsection{Long-Term Contributions}

Based on results like those presented above, it is increasingly understood that California's most severe storms and much of its flood risks derive from land-falling ARs. What is less well recognized is the large extent to which California's overall water resources derive from this same category of storms. Figures 6 and 7 show the precipitation contributions, on a station-by-station basis, from the 1998-2008 SSM/I-derived AR events (Figure 6) and from the 1951-2008 period of the Reanalysis-derived PE events (Figure 7). During the recent SSM/I period (Figure 6), AR storms have contributed anywhere from about 31 to $36 \%$ of overall precipitation at most cooperative weather stations in Central and Northern California, rising to as much as $46 \%$ (Table 3). On average, precipitation during these events amounted to about two to three times the long-term average rates on the same days of year (disregarding AR occurrences). Many ARs making landfall north of California are included in this 
analysis, despite the fact that such storms are less likely to result in major precipitation contributions in California. Nonetheless the landfall of all ARs anywhere on the West Coast are found to have contributed between 30 and $45 \%$ of all precipitation in central and northern California. Fractions of total precipitation in Oregon and Washington deriving from landfalling ARs are similar at many, but not all, stations to these California fractions. When only AR episodes that made landfall in California are considered (not shown here), the fractions contributed remain roughly 30 to $45 \%$ of all precipitation in California because ARs making landfall farther north contribute little precipitation in California so that results are almost the same with or without them.

Figure 6. Contributions of precipitation during wet-season (November-April) days on which atmospheric rivers made landfall on the West Coast (based on Table 1) to overall precipitation from water year 1998 through 2008 at cooperative weather stations in the western US. Inset map shows the ratio of average precipitation on the AR days (including concurrent day and following day) to climatological means for the same combination of days. Concurrent and one following day are composited here to allow for a 1-day uncertainty between the GMT-based AR chronology and local-time cooperative precipitation observations.

CONTRIBUTIONS OF ALL AR EPISODES (days 0 to +1 )

TO TOTAL PRECIPITATION, WY 1998-2008

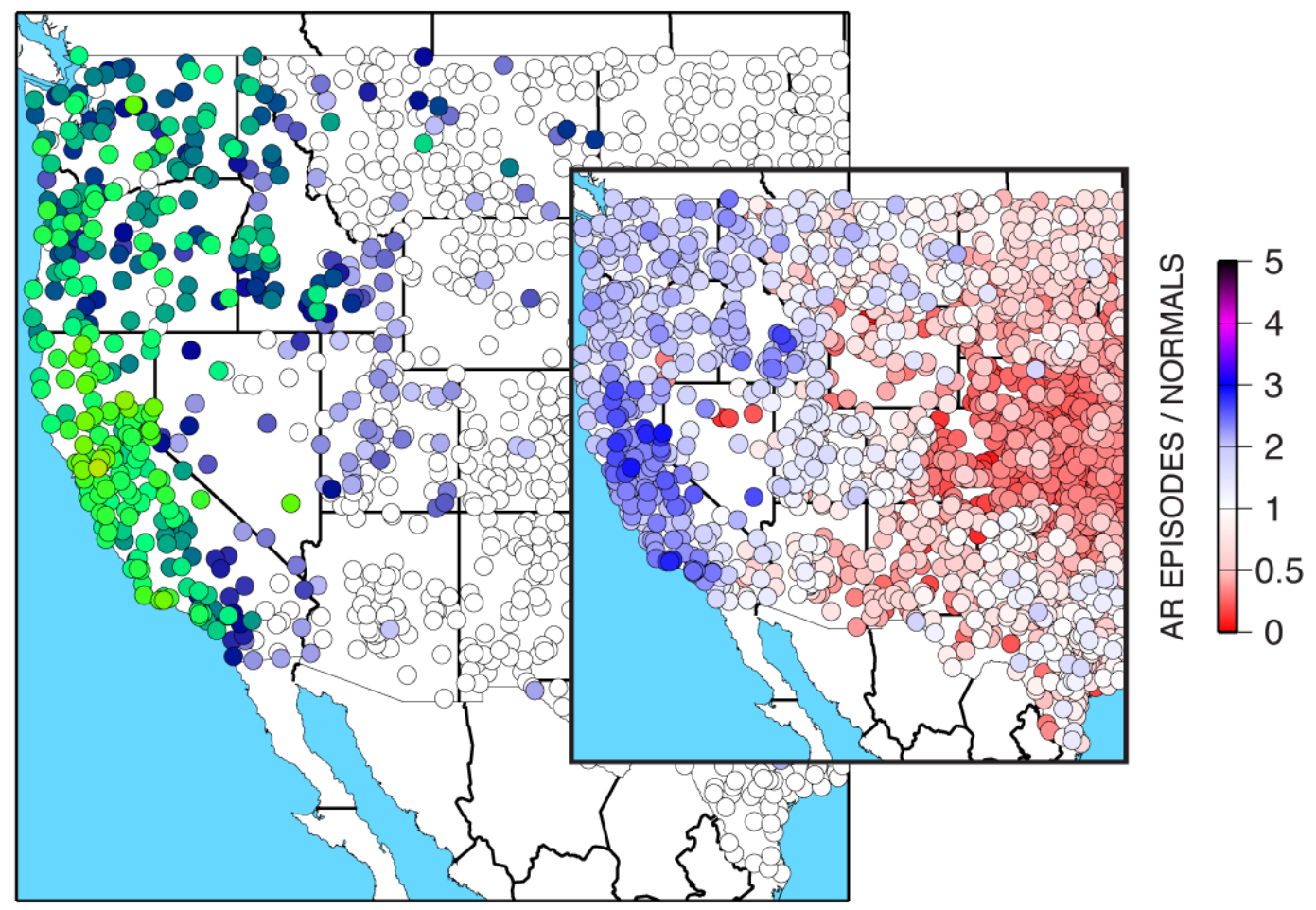

PERCENTAGE OF TOTAL

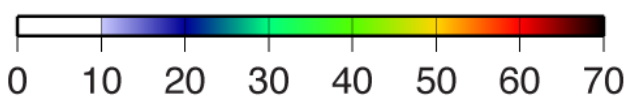


Table 3. Ratios of precipitation and streamflow or runoff during AR and PE events, and subsequent days (as indicated), to overall precipitation, streamflow or runoff, among cooperative precipitation sites and VIC-gridded precipitation data, and streamflow or runoff, among HCDN streamflow gauging stations and gridded VIC simulations, respectively, in central and northern California (east of $120^{\circ} \mathrm{W}$ and south of $42^{\circ} \mathrm{N}$ ). See section 2 for descriptions of data sources.

\begin{tabular}{|c|c|c|c|c|c|c|}
\hline & \multicolumn{2}{|c|}{$\begin{array}{l}\text { Maximum ratio among central and } \\
\text { northern California sites and grid } \\
\text { cells shown in Figures } 6-8\end{array}$} & \multicolumn{2}{|c|}{$\begin{array}{l}\text { 75th-percentile ratio among central } \\
\text { and northern California sites and } \\
\text { grid cells shown in Figures 9-10 }\end{array}$} & \multicolumn{2}{|c|}{$\begin{array}{l}\text { 25th-percentile ratio among central } \\
\text { and northern California sites and } \\
\text { grid cells shown in Figures 9-10 }\end{array}$} \\
\hline & AR events & PE events & AR events & PE events & AR events & PE events \\
\hline & \multicolumn{6}{|c|}{ Precipitation (days 0 and +1 ) } \\
\hline $\begin{array}{l}\text { Cooperative } \\
\text { precipitation } \\
\text { observations }\end{array}$ & $46 \%$ & $22 \%$ & $36 \%$ & $17 \%$ & $31 \%$ & $13 \%$ \\
\hline \multirow[t]{2}{*}{$\begin{array}{l}\text { VIC-gridded } \\
\text { precipitation }\end{array}$} & $45 \%$ & $21 \%$ & $40 \%$ & $16 \%$ & $33 \%$ & $12 \%$ \\
\hline & \multicolumn{6}{|c|}{ Streamflow or Runoff (days 0 to +3 days) } \\
\hline $\begin{array}{l}\text { HCDN } \\
\text { streamflow }\end{array}$ & $71 \%$ & $55 \%$ & $52 \%$ & $38 \%$ & $34 \%$ & $25 \%$ \\
\hline $\begin{array}{l}\text { VIC-simulated } \\
\text { runoff }\end{array}$ & $61 \%$ & $40 \%$ & $43 \%$ & $24 \%$ & $26 \%$ & $12 \%$ \\
\hline
\end{tabular}

During the longer term Reanalysis period (Figure 7), PE storm contributions have amounted to 13 to $17 \%$ of overall precipitation at most stations in central and northern California (Table 3) with the fractions rising to as much at $22 \%$ at the most heavily influenced site, about half the fractions shown in Figure 6. Recall, however, that there are 2.5 times as many ARs per season as PEs, in the chronologies used here, so that the contributions from ARs and PEs are roughly comparable on a storm-by-storm basis. Precipitation from the PE storms average about two to four times the long-term average precipitation rates. To verify the patterns shown, and to fill in spatially (based on the interpolation schemes of [16]), Figure 8 and Table 3 show the corresponding contributions from ARs, water years 1998-2008, and PEs, 1948-2008, as indicated by the gridded VIC-input fields of precipitation. Overall, Figures 6-8 suggest that, whether ARs are considered for a short recent period or PEs for a longer historical period, similar contributions to overall precipitation, on a per-storm basis, are indicated. Thus the large contributions to overall precipitation associated with ARs in the recent $(\mathrm{SSM} / \mathrm{I})$ period are unlikely to be somehow unusual over the longer historical period. 
Figure 7. Same as Figure 6, except for PE days from water year 1951-2008, based on Table 2.

CONTRIBUTIONS OF ALL PE EPISODES (days 0 to +1)

TO TOTAL PRECIPITATION, WY 1951-2008

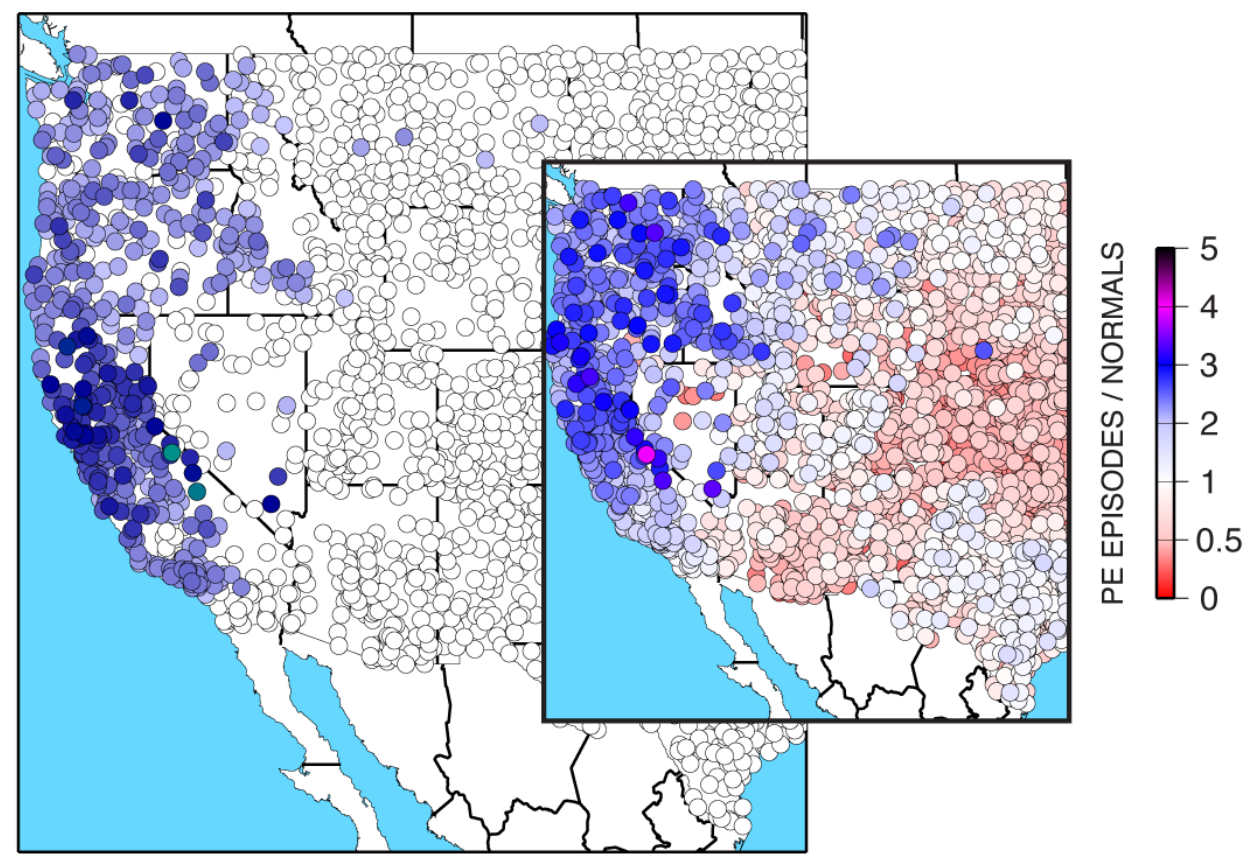

PERCENTAGE OF TOTAL

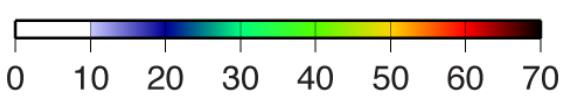

Figure 8. Same as main map in Figure 6, except based on 1/8-degree gridded VIC-input precipitation fields, for (a) AR events and (b) PE events.

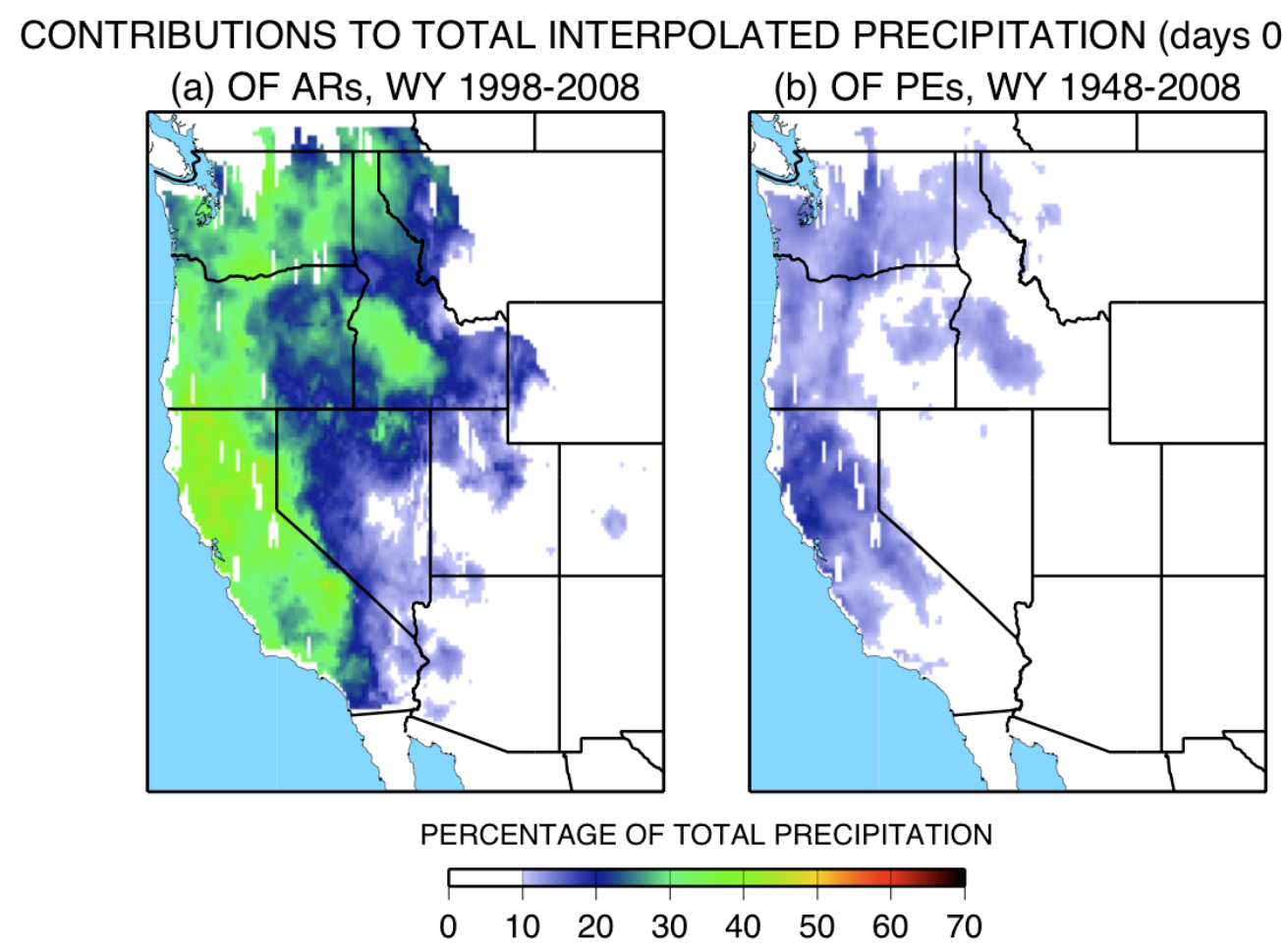


Corresponding analyses of streamflow observations and simulations of runoff-plus-baseflow are presented in Figures 9-10. The wet season along the West Coast is also the cool season so that much precipitation falls as snow, especially in the Sierra Nevada mountains along the eastern edge of Central California. This tendency towards snowfall rather than rain is important because the Sierra Nevada receives many of the highest precipitation totals in the State and generates many of the largest streamflow contributions. As a consequence, much of the precipitation associated with ARs and PEs is stored in seasonal snowpacks until much later in the year and thus it is not possible to catalog precisely as runoff what is from AR or non-AR events using the method here. As an alternative, Guan et al. [28] documented the contribution of AR days to snow water equivalent at snow pillow sites in the Sierra Nevada during WY 2004-2010. Their analysis concluded that on average 6-7 AR events occurred per year, and that they provided $40 \%$ of the total snow water equivalents over the 7 years studied, in broad agreement with the contributions to precipitation documented above. However, low- to mid-altitude parts of the Sierra Nevada and other mountain ranges of California often receive rain from cool-season ARs and from PEs so that significant runoff and streamflow signatures are nonetheless closely associated with AR and PE episodes in river basins across much of the State. When a few days of delay, following the AR or PE episodes, are allowed in calculations of streamflow contributed by these episodes, significant AR and PE contributions to overall streamflow are indicated, with $25-75 \%$ of the sites falling in the range of $34-52 \%$ of streamflow (Table 3 ).

In the SSM/I period, ARs (and a three-day recovery period) are found to yield $50 \%$ and more of overall streamflow in coastal basins from California to the Canadian border (Table 3; Figure 9a). Farther inland, in the Central Sierra Nevada and in the Peninsular Range of southern California, some rivers yield 30 to $50 \%$ of overall streamflow, with most rivers in central and northern California yielding between from about 34 to $52 \%$ of overall streamflow during the AR-influenced periods. These AR streamflows average about two times the long-term mean streamflow rates from similar days of year (not shown). VIC-simulated runoff rates associated with AR episodes amount to broadly similar percentages of overall runoff in coastal basins and in the mountains of California (Figure 10a). Significant fractional contributions to simulated runoff actually extend considerably father inland than in the observed streamflows. However, much of the area with the largest fractional contributions to overall runoff are areas that do not contribute much overall runoff at all, in absolute terms; AR runoff from such areas does not add to overall streamflows much and thus do not show up in the measured river discharges. Therefore, contributions mapped in Figure 10 were masked to exclude all areas where average AR runoff generation was less than $0.5 \mathrm{~mm} /$ day. With this masking, comparisons between observed streamflow contributions from ARs and simulated runoff contributions are in good geographic agreement, although simulated fractional runoff contributions are generally smaller than their nearby streamflow counterparts. 
Figure 9. Contributions of streamflow during wet-season (November-April) episodes when atmospheric rivers (as ARs and PEs) made landfall on the West Coast to overall streamflow at HCDN streamflow gauging stations [14] in the western US, for (a) AR episodes, water years 1998-2008, based on Table 1, and (b) PE episodes, water years 1949-2008, based on Table 2 (including streamflow in each case from PE-concurrent day and three following days). Inset map shows the ratio of average streamflow on the PE days (including concurrent day and 3 following days) to climatological means for the same combination of days. Concurrent and three following days are considered to allow for streamflow response times from the basins. Nonetheless, because higher-altitude mountains, such as the Sierra, capture large amounts of snow during AR or PE events that does not runoff until spring or summer, runoff percentages from these regions are expected to be underestimates overall.

CONTRIBUTIONS TO TOTAL STREAMFLOW

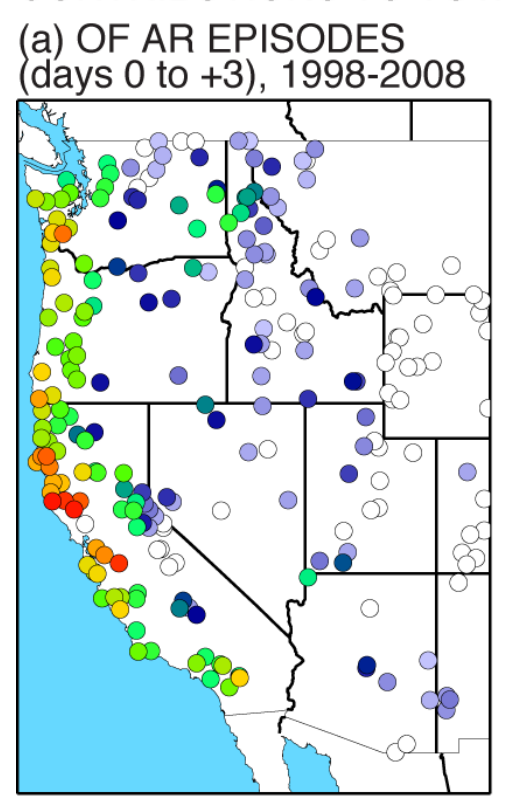

(b) OF PE EPISODES

(days 0 to +3), 1949-2008

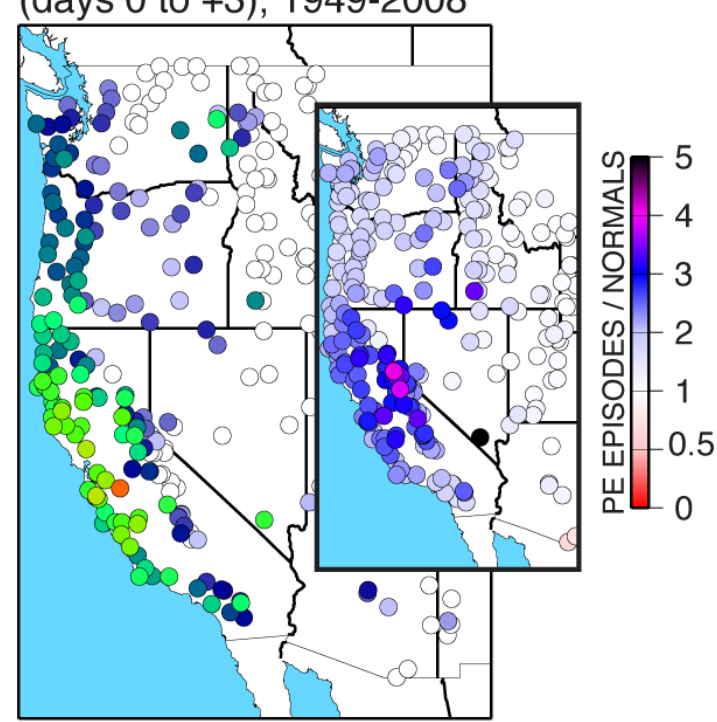

PERCENTAGE OF TOTAL

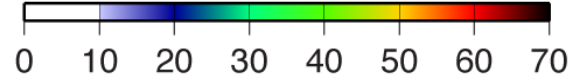


Figure 10. Same as main map in Figure 8, except based on VIC-simulated runoff-plus-baseflow, and for (a) AR episodes and (b) PE episodes; both maps are masked to show AR- (or PE-) episode contributions to runoff-plus-baseflow in areas where average simulated runoff-plus-baseflow during episodes is $>0.5 \mathrm{~mm} /$ day.

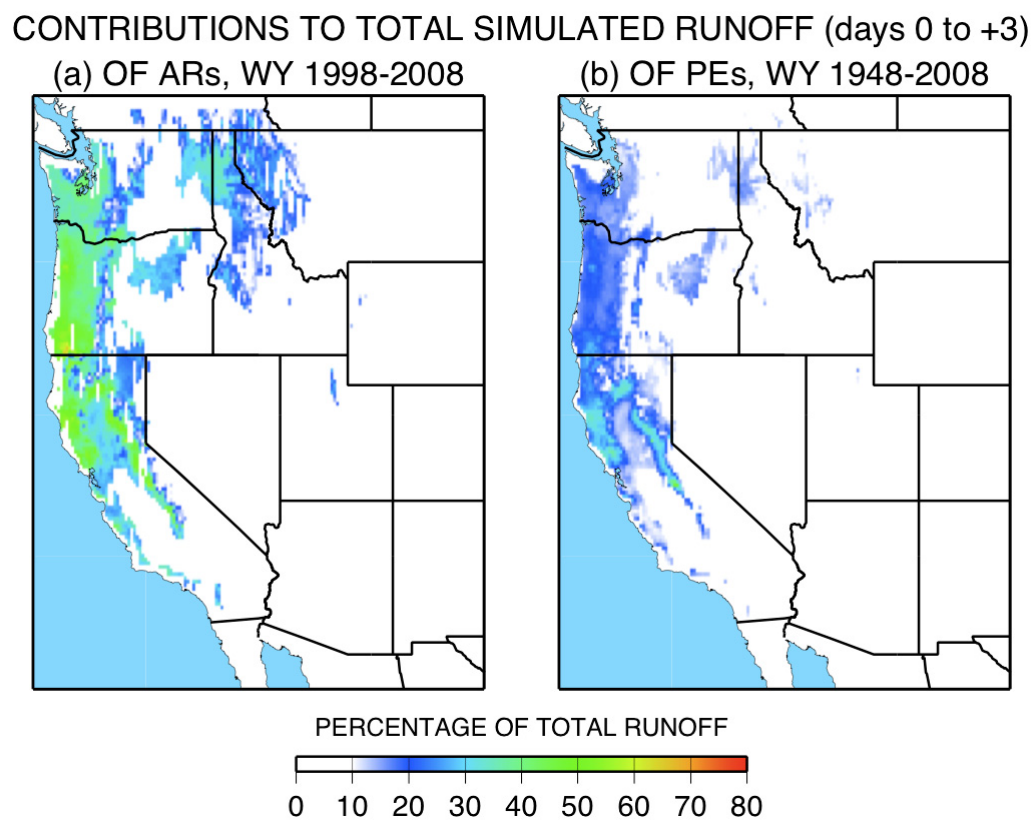

On the longer term, streamflow yields associated with the PE episodes from water year 1949-2009 (Figure $9 \mathrm{~b}$ and Table 3) are somewhat less than the AR contributions (Figure 9a) in California, because there are about 2.5 times as many AR episodes per year as PE episodes. The streamflow contributions from PEs in the rivers of the Pacific Northwest are, similarly, about half as large as the (percentage) contributions from the AR episodes. Simulated runoff associated with PEs (Figure 10b) approximates but is smaller than the AR contributions (Figure 10a) in mountainous areas of northern California, and are much smaller in the Pacific Northwest.

Overall then, significant fractions of all precipitation and streamflow in the West Coast states derive from AR storms. This observation, together with the fact that a notably few storms (most often, AR storms) contribute most of California's precipitation each year, on average, and that California's year-to-year precipitation (and streamflow) variability is quite large, makes increased understanding of the details of how, where and when ARs arrive to support the State's water resources crucial.

\subsection{Year-To-Year Contributions}

Contributions of PE events to water year precipitation (and streamflow) vary from year to year. Average PE contributions to water-year precipitation at cooperative weather stations in central and northern California are shown in Figure 11, with contributions ranging from zero in several years to as much as $54 \%$ in 1986 . To what extent do these variations (and variations elsewhere along the west coast) reflect large-scale climate modes either as concurrent or predictive associations?

Long-term, reproducible influences of large-scale Pacific climate influences on the occurrence of PEs, in general, remain difficult to discern or predict at present [5], although on an event-by-event 
basis, strong ties to the tropical Pacific have been documented [11,29]. Nonetheless, year-to-year phases [30] of the interannual El Nino-Southern Oscillation (ENSO) climate mode in the tropical Pacific [31] and of the multidecadal Pacific Decadal Oscillation (PDO, [32]) mode in the North Pacific climate are significantly associated with increases in the PE fractions of water-year precipitation (Figure 12). The association indicated is such that, in El Nino and positive PDO (El Nino-like) years, PE fractions of water-year precipitation at stations in southern and south-central California, and, more spottily, in southern California and western Washington, respectively, are significantly larger than average. Comparison of Figure 12 with Figure 7 suggests that the correlations in Figure 12 have their largest practical impacts in south central California and, perhaps, Washington, where both (a) the correlations with ENSO and PDO are significant and (b) the fractions of precipitation associated with PEs are also large. Notably, significant connections between ENSO or PDO and PE-precipitation contributions are not found in central and northern California from which so most of the State's water resources are generated. Although such relations may eventually support improvements in long-lead forecasts of the role that PEs and floods will play in the overall water resources of southern California and western Washington, the simple (concurrent) relations in Figure 12 could not be extended backwards in time into significant predictive associations between the status of ENSO or PDO in summer with PE-precipitation contributions at western weather stations.

Figure 11. Averages of contributions to water-year total precipitation on PE days (plus day +1 ) at 202 cooperative weather stations in central and northern California (same area as defined in caption of Table 3), 1951-2008.

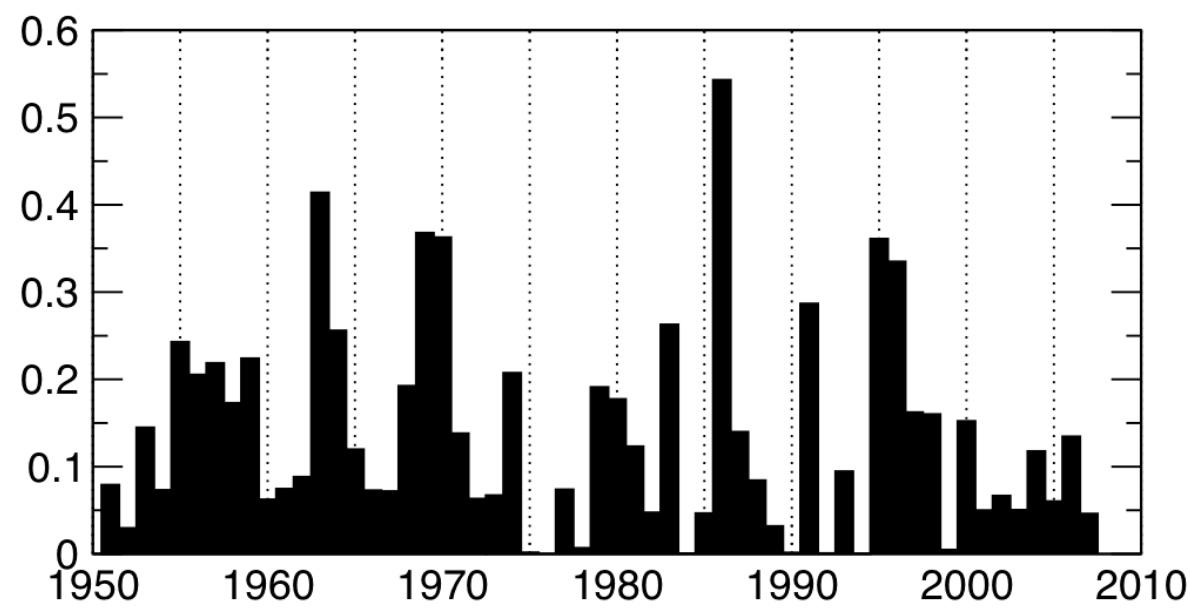


Figure 12. Correlations between PE contributions to water-year precipitation totals and concurrent water-year averages of (a) the Nino3.4 [31] sea-surface temperature (SST) index of the tropical ENSO climate mode, averaging SSTs in the region $5^{\circ} \mathrm{N}-5^{\circ} \mathrm{S}$, $120^{\circ} \mathrm{W}-170^{\circ} \mathrm{W}$, and (b) the Pacific Decadal Oscillation SST-based index [32]; colored dots indicate cooperative weather stations where the correlations are significantly different from zero at $95 \%$ confidence level and small gray dots are stations where the relations are not significant at this level.

\section{CORRELATIONS OF AR PRECIPITATION (days 0 to +1) CONTRIBUTIONS WITH:}
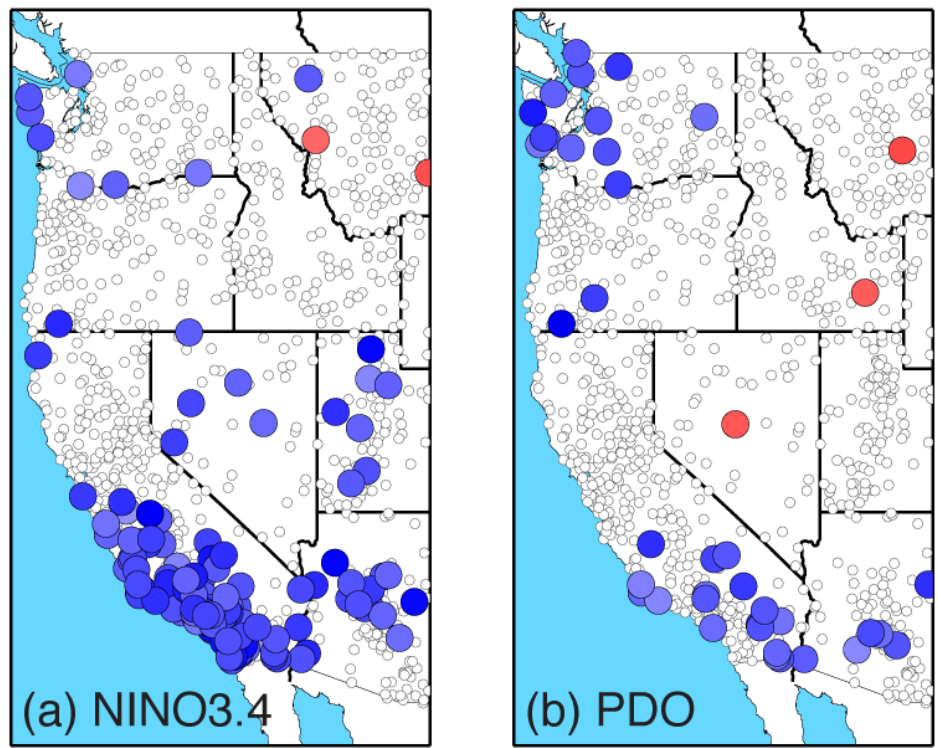

Correlation, as percentage

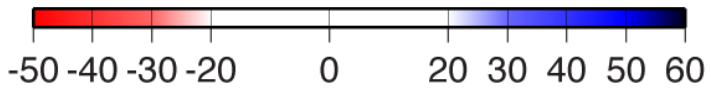

As noted in Figure 12, no reliable associations exist between ENSO or PDO status and PE-contributions to water-year precipitation in central and northern California, but this part of California lies along transition zones of many ENSO and PDO hydroclimatic influences [33,34], so that reliable teleconnections of those modes to northern California hydroclimatic conditions do not generally occur. Thus, in order to identify concurrent or predictive climate associations with PE-precipitation contributions in central and northern California, a wider range of possibilities must be investigated. In Figure 13, rank correlations between the time series in Figure 12 and concurrent (a) global sea-surface temperatures and (b) Pacific-North American 700-mbar heights are mapped. Above the planetary boundary layer, the large-scale atmospheric flow is nearly in geostrophic balance, and thus lines of equal height to the 700 mbar pressure surface approximate closely the stream lines followed by winds at the same level. Correlations with 700-mbar heights also indicate areas where the passage of low- and high-pressure weather systems are associated with the PE contributions.

Correlations are only mapped in Figure 13 where they are significantly different from zero correlations with a simple student-t test. Thus the most significant correlations between winter sea-surface temperatures and PE-precipitation contributions in central and northern California 
(Figure 13a) are found in the westernmost Pacific, with positive correlations (red) with sea-surface temperatures northeast of Japan and negative correlations (blue) in the tropical Pacific near the Philippines and Indonesia. A dipole of correlations is also found in the tropical and subtropical Atlantic basin north of the equator. The pattern of positive sea-surface temperature correlations in the western Pacific is located just north of the Kuroshio Extension Current and just north of the strongest part of the PDO sea-surface temperature pattern ([32], and outlined in green in Figure 13a). Thus the Pacific sea-surface temperature correlations with PE-precipitation contributions in central and northern California straddle (but do not parallel) the canonical PDO pattern and thus may reflect connections between large-than-average PE-precipitation contributions and concurrent southward displacements of the canonical positive-PDO sea-surface temperature pattern. Similarly, correlations between the PE-precipitation contributions and 700-mb heights (Figure 13b) appear to indicate increased PE contributions in winters with lower-than-average 700-mbar heights or southward displacements of the climatological Aleutian Low pressure pattern (beneath the 700-mb center of action for PDO is indicated in green in Figure 13b) [33], which in turn reflects enhanced passage of low-pressure systems and storms across the North Pacific basin. Thus the 700-mb height correlations in Figure $13 \mathrm{~b}$ suggest a tendency for large PE-precipitation contributions in central and northern California to be associated with southern displacements of the storm tracks over the midlatitude Pacific and enhancement of the subtropical jet over the North Pacific basin (not shown). In particular, the structure of the low $700 \mathrm{mb}$ height anomalies indicated by the negative corrections positioned north of Hawaii is consistent with atmospheric circulations that would favor northward advection of water vapor from the tropics near Hawaii - a classic characteristic of PE-type AR events [5,29].

Notably, no significant sea-surface temperature correlations are indicated in the equatorial central or eastern Pacific areas associated with tropical ENSO variability, and the only significant ties to equatorial sea-surface temperatures appear in the far westernmost parts of the Tropical Pacific "warm pool" around the Philippines. This warm pool is location of some of the warmest sea-surface temperatures on the planet, is a source of some of the warm waters associated with El Niños, and perhaps most importantly is a region that has seen important multi-decadal warming trends since the 1970s [35]. The negative (blue) correlations indicated in this area would, on long-term average, suggest that warming of the Pacific warm pool since the 1970s may have contributed to the broad declines in PE contributions from the 1980s to 2008 in Figure 11 (in agreement with the North American precipitation teleconnections found in [35]), and that continued warming might be associated with decreasing contributions of PEs to California precipitation, if the historical associations continue. 
Figure 13. Rank correlations between average PE contributions to water-year precipitation totals at 202 cooperative weather stations in central and northern California (same area defined in Table 3) and concurrent (a) November-April sea-surface temperatures (SSTs), and (b) November-April 700 mbar height anomalies [12]; green hatched shapes in North Pacific surround primary centers of action (largest correlations) with respect to Pacific Decadal Oscillation [31].

\section{a) RANK CORRELATIONS WITH SSTS}

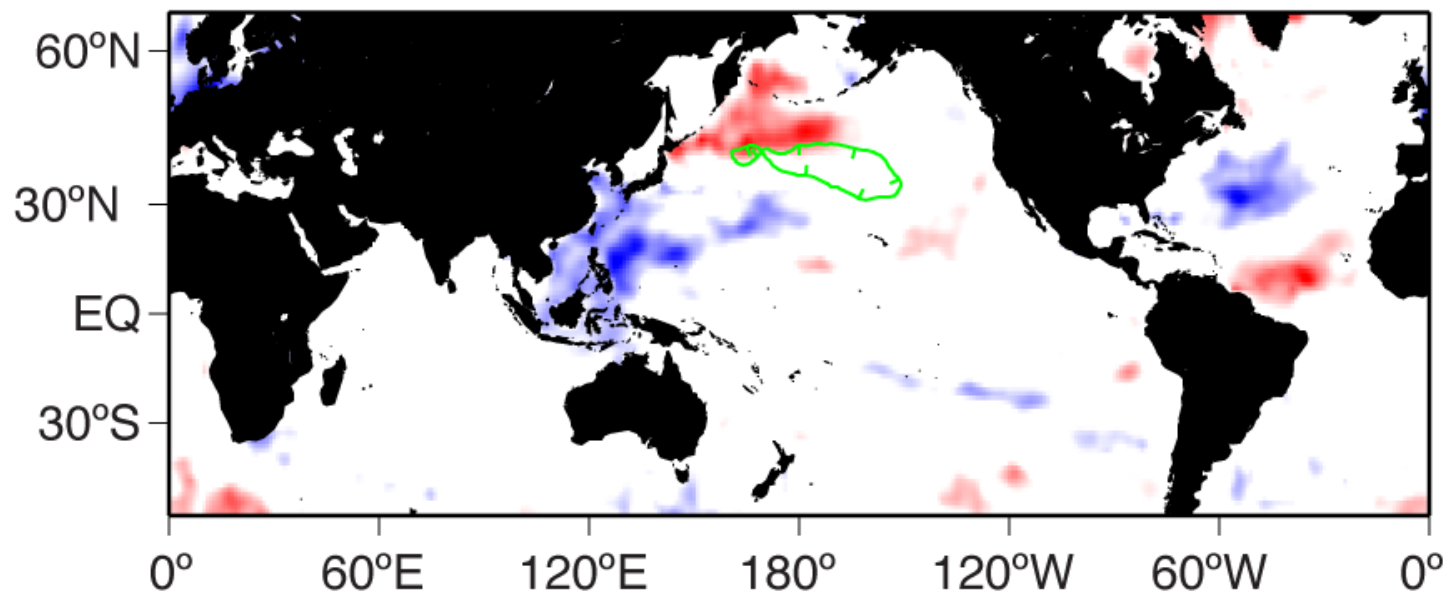

b) RANK CORRELATIONS WITH 700mb HEIGHTS

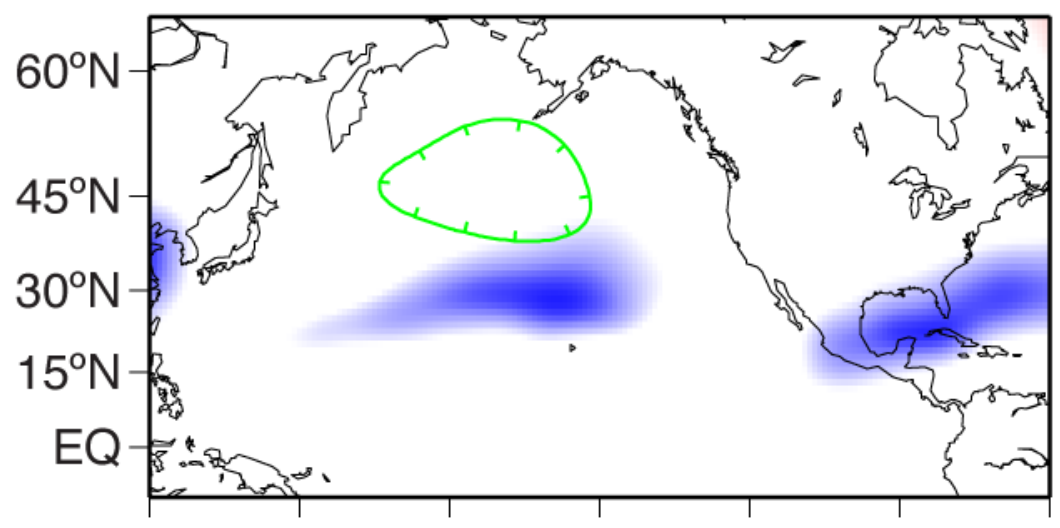

$120^{\circ} \mathrm{E} 150^{\circ} \mathrm{E} 180^{\circ} 150^{\circ} \mathrm{W} 120^{\circ} \mathrm{W} 90^{\circ} \mathrm{W} 60^{\circ} \mathrm{W}$

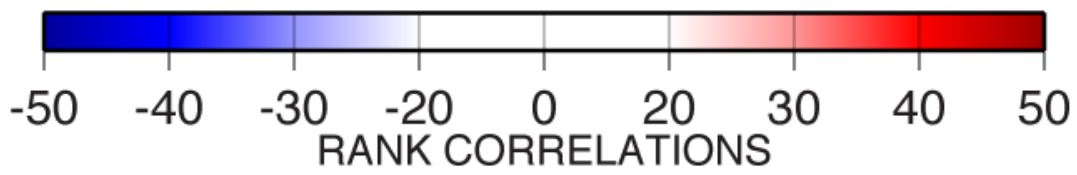

Finally, historical relations between climatic conditions at the beginning of a water year and the PEprecipitation contributions in central and northern California during that water year are explored in Figure 14. The correlations between sea-surface temperatures at the start of a water year and PE contributions (Figure 14a) indicate that the connections between Pacific (and, in this case, Indian Ocean) warm pool and California PE-precipitation contributions may have some predictive elements. Cooler-than-normal warm pool sea-surface temperatures have historically tended to be associated with larger-than-normal PE contributions. As with the concurrent correlations in Figure 13a, no other equatorial connection is apparent. Intriguingly, an impressive, if difficult to understand, historical 
connection appears, in the three panels of Figure 14, that associates unusually wet conditions in the Pacific Northwest (14c), unusually low offshore pressures (14b) and underlying slightly cool sea-surface temperatures (14a) at the beginning of a water year (September-October) with higher than normal PE-precipitation contributions for central and northern California during the rest of the water year.

Figure 14. Rank correlations between average PE contribution to water-year precipitation totals at 202 cooperative weather stations in central and northern California (same area defined in Table 3) and (a) preceding September-October sea-surface temperatures (SSTs), (b) preceding September-October 700 mbar height anomalies, and (c) preceding September-October precipitation [12].

a) RANK CORRELATIONS WITH SSTS

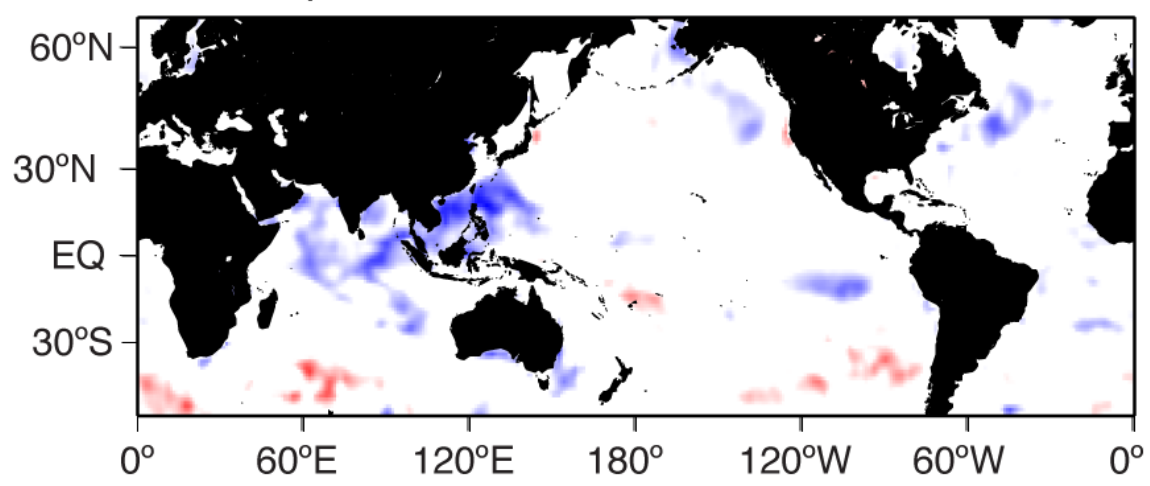

b) RANK CORRELATIONS WITH 700mb HEIGHTS

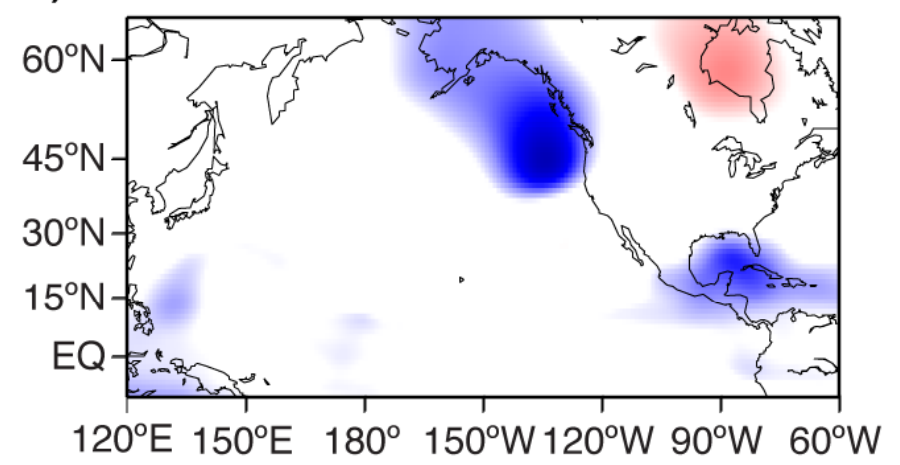

c) RANK CORRELATIONS WITH PRECIPITATION
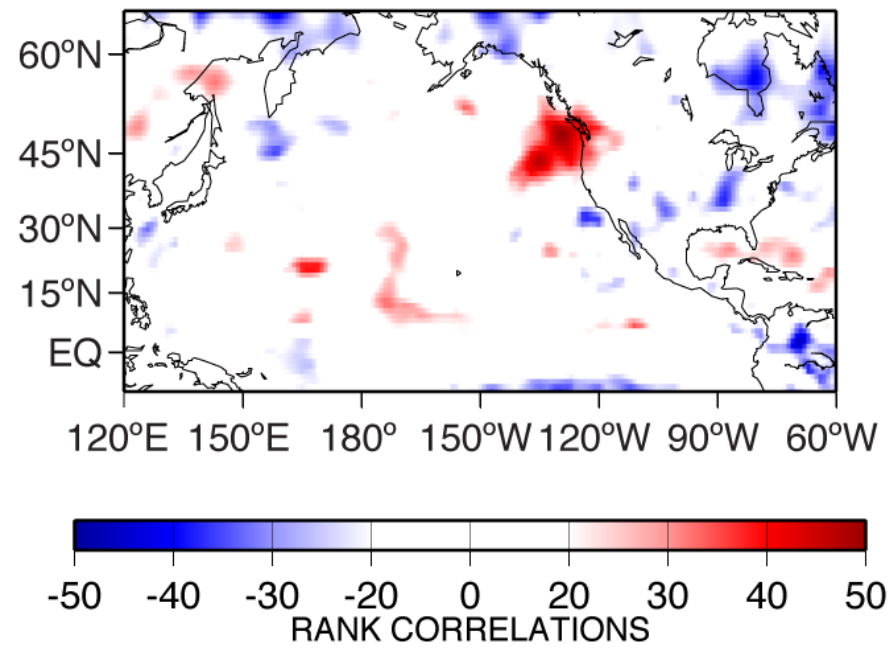
In principle, such relations might be used to condition the expectations of decision makers or to explicitly predict water years in which California will receive above-normal fractions of its overall precipitation and surface water supplies from PE storms. In such years, tradeoffs between managing reservoirs during major storms to focus on the hazardous aspects of these storms rather than their contributions to overall water resources might be particularly difficult, as the PE contributions are large and their forfeiture to flood-management practices particularly painful to resource managers. In years when PE storms will provide smaller fractions of the overall resources, more aggressive flood-management strategies during PE episodes might be implemented with greater confidence that the overall water resource will still fare well.

\section{Summary and Conclusions}

Nationwide analyses of precipitation records from long-term cooperative weather stations, together with corresponding analyses of streamflow records from the HCDN network, reveals the remarkable interannual variability of California's precipitation and streamflows. This variability derives in part from the relatively few storms that contribute the bulk of California's precipitation each year, on average. When a few storms either don't arrive or "underperform", the precipitation totals and thus water resources in the State suffer. The capacity of a relatively few storms each year to support California's water resources owes much to the extreme precipitation that some of its storms yield, and we find that, in recent decades, landfalling atmospheric rivers (ARs) have caused most of the largest of these storms. ARs generally, as well as in a particularly well known but not notably more or less intense subset of ARs called pineapple-express storms (PEs), bring intense and warm precipitation to the mountain catchments of California. The disproportionately large contributions made by ARs are a primary meteorological factor in flood generation on many California rivers, as well as a primary source of precipitation and water resources in the State. On long-term average, AR storms contribute from $20-50 \%$ of the state's precipitation totals, with area-average contributions ranging from zero to $54 \%$ from year to year, historically. The storms result in comparable fractions of overall streamflow in much of the State. With respect to these year-to-year differences, correlations of the year-to-year differences in the fractions contributed in river basins along the west coast and large-scale ENSO, PDO, sea-surface temperatures, and atmospheric conditions suggest that it may be possible to forecast some of these fluctuations on interseasonal time scales.

Thus we find that close integration of water-resource and flood management actions and strategies may rightly be motivated by more than a need for efficiency or for eking a little more water supply out of a relatively dry landscape in the face of growing demands. Instead the need for such integration has a firm physical basis in the shared meteorology of water resources and floods in California. Both water resources and floods in California are strongly dependent on the character and frequency of landfalling AR storms arriving from over the North Pacific Ocean. Understanding of these storms has grown dramatically in recent years, but—in view of their crucial, dual role — continued and expanded research and monitoring are needed, focusing specifically on ARs and the contributions to water resources and to hazards that they yield. Of particular significance, the distributions of vapor transports, and thus precipitation, once the ARs are over land and interacting with orography need to be better understood and predicted. AR storms may also be key to determining and predicting changes of both California's 
water resources and flood regimes under projected global climate changes. At present, the fate of ARs has only begun [36] to be explored in climate-change projections. More attention to the possible influences of greenhouse-gas-induced climate changes on AR frequencies and magnitudes, whether within modern climate models or from more basic physical principles, is needed.

\section{Acknowledgments}

The estimates of integrated water-vapor transports used for the most recent PE chronology here were kindly provided by Cathy Smith of the NOAA Earth Systems Research Laboratory. This work was conducted in the context of CalWater atmospheric-river research efforts [37] organized by the California Energy Commission, National Oceanic and Atmospheric Administration, U.S. Geological Survey, and Scripps Institution of Oceanography.

\section{References}

1. California Department of Water Resources (DWR). California Water Plan Update-A Framework for Action-DWR Bulletin 160-05; DWR: Sacramento, CA, USA, 2005; p. 24. Available online: http://www.waterplan.water.ca.gov/docs/cwpu2005/cwphighlights/highlights.pdf.

2. Kelley, R. Battling the Inland Sea-Floods, Public Policy and the Sacramento Valley; University of California Press: Berkeley, CA, USA, 1998; p. 420.

3. Weaver, R.L. Meteorology of Hydrologically Critical Storms in California; U.S. Department of Commerce Hydrometeorology Report No. 37; Department of Commerce Hydrometeorology: Washington, DC, USA, 1962; p. 207.

4. Higgins, R.W.; Schemm, J.K.E.; Shi, W.; Leetmaa, A. Extreme precipitation events in the western United States related to tropical forcing. J. Climate 2000, 13, 793-820.

5. Dettinger, M.D. Fifty-Two Years of Pineapple-Express Storms across the West Coast of North America; California Energy Commission PIER Energy-Related Environmental Research Report CEC-500-2005-004; California Energy Commission: Sacramento, CA, USA, 2004; p. 15.

6. Zhu, Y.; Newell, R.E. A proposed algorithm for moisture fluxes from atmospheric rivers. Mon. Wea. Rev. 1998, 126, 725-735.

7. Ralph, F.M.; Neiman, P.J.; Wick, G.A. Satellite and CALJET aircraft observations of atmospheric rivers over the eastern North-Pacific Ocean during the winter of 1997/98. Mon. Wea. Rev. 2004, 132, 1721-1745.

8. Neiman, P.J.; Ralph, F.M.; Wick, G.A.; Kuo, Y.H.; Wee, T.K.; Ma, Z.; Taylor, G.H.; Dettinger, M.D. Diagnosis of an intense atmospheric river impacting the Pacific NorthwestStorm summary and offshore vertical structure observed with COSMIC satellite retrievals. Mon. Wea. Rev. 2008, 136, 4398-4420.

9. Ralph, F.M.; Neiman, P.J.; Rotunno, R. Dropsonde observations in low-level jets over the northeastern Pacific Ocean from CALJET-1998 and PACJET-2001: Mean vertical-profile and atmospheric-river characteristics. Mon. Wea. Rev. 2005, 133, 889-910.

10. Ralph, F.M.; Neiman, P.J.; Wick, G.A.; Gutman, S.; Dettinger, M.; Cayan, D.; White, A.B. Flooding on California's Russian River-Role of atmospheric rivers. Geophys. Res. Lett. 2006, 33,5 . 
11. Bao, J.W.; Michelson, S.A.; Neiman, P.J.; Ralph, F.M.; Wilczak, J.M. Interpretation of enhanced integrated water vapor bands associated with extratropical cyclones: Their formation and connection to tropical moisture. Mon. Wea. Rev. 2006, 134, 1063-1080.

12. Kalnay, E.; Kanamitsu, M.; Kistler, R.; Collins, W.; Deaven, D.; Gandin, L.; Iredell, M.; Saha, S.; White, G.; Woollen, J.; Zhu, Y.; Chelliah, M.; Ebisuzaki, W.; Higgins, W.; Janowiak, J.; Mo, K.C.; Ropelewski, C.; Wang, J.; Leetmaa, A.; Reynolds, R.; Jenne, R.; Joseph, D. The NCEP/NCAR 40-year reanalysis project. Bull. Amer. Meteor. Soc. 1996, 77, 437-471.

13. National Weather Service (NWS). National Weather Service Observing Handbook Number 2. Cooperative Station Observations; Observing Systems Branch, Office of Systems Operations: Washington, DC, USA, 1989; p. 94. Available online: http://www.nws.noaa.gov/om/ coop/Publications/coophandbook2.pdf (accessed on 3 February 2010).

14. Slack, J.R.; Landwehr, J.M. Hydro-climatic Data Network (HCDN): A U.S. Geological Survey Streamflow Data Set of the United States for the Study of Climate Variations, 1874-1988; USGS Open-File Report 92-129; U.S. Geological Survey (USGS): Reston, VA, USA, 1992; p. 193.

15. Liang, X.; Lettenmaier, D.P.; Wood, E.F.; Burges, S.J. A simple hydrologically based model of land surface water and energy fluxes for GSMs. J. Geophys. Res. 1994, 99, 14415-14428. (Version 4.0.6 used here).

16. Hamlet A.F.; Lettenmaier, D.P. Production of temporally consistent gridded precipitation and temperature fields for the continental U.S. J. Hydrometeor. 2005, 6, 330-336. Available online: http://www.hydro.washington.edu (accessed on 12 November 2010).

17. Daly, C.; Neilson, R.P.; Phillips, D.L. A statistical-topographic model for mapping climatological precipitation over mountainous terrain. J. Appl. Meteor. 1994, 33, 140-158.

18. Durrre, I.; Menne, M.J.; Gleason, B.E.; Houston, T.G.; Vose, R.S. Comprehensive automated quality assurance of daily surface observations. J. Appl. Meteorol. Clim. 2010, 49, 1615-1633.

19. Wood, A.W.; Lettenmaier, D.P. A testbed for new seasonal hydrologic forecasting approaches in the western US. Bull. Amer. Meteorol. Soc. 2006, 87, 1699-1712.

20. Maurer, E.P.; Wood, A.W.; Adam, J.C.; Lettenmaier, D.P.; Nijssen, B. A long-term hydrologically-based data set of land surface fluxes and states for the conterminous United States. J. Climate 2002, 15, 3237-3251.

21. Wood, E.F.; Lettenmaier, D.P.; Zartarian, V.G. A land-surface hydrology parameterization with subgrid variability for general circulation models. J. Geophys. Res. 1992, 97, 2717-2728.

22. Neiman, P.J.; Ralph, F.M.; Wick, G.A.; Lundquist, J.; Dettinger, M.D. Meteorological characteristics and overland precipitation impacts of atmospheric rivers affecting the West Coast of North America based on eight years of SSM/I satellite observations. J. Hydrometeor. 2008, 9, $22-47$.

23. Hollinger, J.P.; Peirce, J.L.; Poe, G.A. SSM/I instrument evaluation. IEEE Transactions on Geosci. Remote Sens. 1990, 28, 781-790.

24. Wentz, F.J. The Intercomparison of 53 SSM/I Water Vapor Algorithms; Remote Sensing Systems Technical Report on WetNet Water Vapor Intercomparison Project (VIP); Remote Sensing Systems: Santa Rosa, CA, USA, 1995; p. 19. 
25. Cayan, D.R.; Dettinger, M.D.; Redmond, K.T.; McCabe, G.J.; Knowles, N.; Peterson, D.H. The transboundary setting of California's water and hydropower systems-Linkages between the Sierra Nevada, Columbia River, and Colorado River hydroclimates. In Climate and WaterTransboundary Challenges in the Americas; Diaz, H.F., Morehouse, B.J., Eds.; Kluwer Academic: Dordrecht, The Netherlands, 2003; pp. 237-262.

26. Ralph, F.M.; Dettinger, M.D. Extreme precipitation patterns across the contiguous United States documented using 3-day rainfall categories. Bull. Am. Meteor. Soc. Submitted.

27. Jeton, A.E. US Geological Survey Fact Sheet 2006-3102; Flood Chronology of the Carson River Basin, California and Nevada Web Site: Carson City, NV, USA, 2006; p. 2.

28. Guan, B.; Molotch, N.P.; Waliser, D.E.; Fetzer, E.J.; Neiman, P.J. Extreme snowfall events linked to atmospheric rivers and surface air temperature via satellite measurements. Geophys. Res. Lett. 2010, 37, L20401.

29. Ralph, F.M.; Neiman, P.J.; Kiladis, G.N.; Weickmann, K.M.; Reynolds, D.W. A multi-scale observational case study of a Pacific atmospheric river exhibiting tropical-extratropical connections and a mesoscale frontal wave. Mon. Wea. Rev. 2010, in press.

30. McCabe, G.J.; Dettinger, M.D. Primary modes and predictability of year-to-year snowpack variations in the western United States from teleconnections with Pacific Ocean climate. J. Hydrometeor. 2002, 3, 13-25.

31. Trenberth, K.E. The definition of El Niño. Bull. Am. Met. Soc. 1997, 78, 2771-2777.

32. Mantua, N.J.; Hare, S.R.; Zhang, Y.; Wallace, J.M.; Francis, R.C. A Pacific interdecadal climate oscillation with impacts on salmon production. Bull. Am. Met. Soc. 1997, 78, 1069-1079.

33. Redmond, K.T.; Koch, R.W. Surface climate and streamflow variability in the western United States and their relationship to large-scale circulation indices. Water Resour. Res. 1991, 27, 2381-2399.

34. Cayan, D.R.; Webb, R.H. El Nino/Southern Oscillation and streamflow in the western United States. In El Nino: Historical and Paleoclimatic Aspects of the Southern Oscillation; Diaz, H.F., Markgraf, V., Eds.; Cambridge University Press: Cambridge, UK, 1992; pp. 29-68.

35. Wang, H.; Mehta, V.M. Decadal variability of the Indo-Pacific warm pool and its association with atmospheric and oceanic variability in the NCEP-NCAR and SODA Reanalysis. J. Climate 2008, $21,5545-5565$.

36. Dettinger, M.D. Climate change, atmospheric rivers and floods in California-A multimodel analysis of storm frequency and magnitude changes. J. Am. Water Resour. Assoc. in press.

37. More information available online: http://www.esrl.noaa.gov/psd/calwater/ (accessed on 2 January 2011).

(C) 2011 by the authors; licensee MDPI, Basel, Switzerland. This article is an open access article distributed under the terms and conditions of the Creative Commons Attribution license (http://creativecommons.org/licenses/by/3.0/). 